\title{
Preclinical Assessment of Nanostructured Liquid Crystalline Particles for the Management of Bacterial Keratitis: in-vivo and Pharmacokinetics Study
}

Shreya Kaul ( $\sim$ shreyakaul421@gmail.com )

IFTM University https://orcid.org/0000-0002-8540-3612

Upendra Nagaich

Amity University

Navneet Verma

IFTM University

\section{Research Article}

Keywords: Nanostructured liquid crystalline particles, Histopathological studies, HET-CAM test, Ocular pharmacokinetic studies, Bacterial keratitis

Posted Date: May 11th, 2021

DOl: https://doi.org/10.21203/rs.3.rs-481365/v1

License: (c) (1) This work is licensed under a Creative Commons Attribution 4.0 International License.

Read Full License 


\section{Abstract}

The research work was driven to develop novel nanostructured liquid crystalline particles of vancomycin for its improved pre-ocular residence time, ocular bio-availability, enhanced targeting, increased permeability, reduced dosing frequency, controlled drug release and reduced systemic side-effects. Formulation was developed by fragmenting cubic crystalline phase of glycerol monooleate, water and poloxamer 407. A four-factor, three-level Taguchi statistical experimental design was constructed to optimize the formulation. Formulations exhibited internal-cubic structure of the vesicles with particle size in the range of $51.11 \pm 0.96 \mathrm{~nm}$ to $158.73 \pm 0.46 \mathrm{~nm}$ and negative zeta potential. Ex-vivo transcorneal permeation studies demonstrated that the optimized cubosomes had 2.4-fold increase in apparent permeability co-efficient as compared to vancomycin solution. Whereas, in-vivo studies in rabbits demonstrated that the severity of keratitis was considerably lowered in day 3 with optimized cubosomes. Ocular pharmacokinetic studies evaluated level of drug in aqueous humor and results revealed that the time to peak concentration $\left(T_{\max }\right)$ of vancomycin loaded cubosomal formulation was about 1.9-fold higher and mean residence time was 2.2-fold greater than vancomycin solution. Furthermore, histological examination revealed that the corneal layers displayed well-maintained morphology without any stromal swelling, consequently indicating safety of formulation. In conclusion, results manifested that the developed vancomycin loaded cubosomes could be a promising novel ocular carrier and an ideal substitute for conventional eye-drops for the management of bacterial-keratitis.

\section{Introduction}

Glycopeptide antibiotic, vancomycin is a tricyclic-glycosylated non-ribosomal peptide. It is used to treat diverse bacterial infections, specifically gram + ve infections such as species of Staphylococcus. Vancomycin has minimal oral absorption and is delivered by intravenous infusion. It acts by inhibiting polymerization of peptidoglycan layer in cell-wall of bacteria. Specifically, vancomycin averts incorporation of $\mathrm{N}$-acetylmuramic acid-(NAM) and $\mathrm{N}$-acetylglucosamine-(NAG)-peptide sub-units from being incorporated into the peptidoglycan matrix. Vancomycin forms hydrogen bonds with the terminal D-alanyl-D-alanine moieties of the NAM/NAG-peptides, which impedes glucosyltransferase and the Pphospholipid carrier, therefore averting synthesis and polymerization of NAM/NAG-peptide subunits into the peptidoglycan layer. This inhibition weakens the cell-wall of bacteria and causes intracellular contents to leak-out, eventually causing bacterial cell-death [1].

Bacterial keratitis can be described as vision threatening ocular infection predominantly caused by Staphylococcus aureus, Streptococcus pneumoniae and Pseudomonas aeruginosa. It presents clinically with corneal inflammation, pain, excessive tearing, corneal edema, ulcers, reduced vision, redness and photophobia. Pre-disposing factors for instance, extended use of contact lenses, eye-trauma, ocular surface-disorders and dry-eyes are correlated with bacterial keratitis. As these factors might alter the defence-mechanism of ocular-surface and allow bacterias' to invade membrane of cornea. There is break-down of corneal-epithelium due to which there is penetration of bacteria. Following the penetration, 
there is activation of plasminogen to plasmin and bacteria secrets protease, chymase and tryptase that causes corneal necrosis leading to rapid corneal weakening and perforation [2].

Management of bacterial keratitis remains a challenge due to poor corneal penetration of conventional eye-drops and unique anatomical eye-structure. The entry of drug molecule at the site of action is sometimes restricted owing to the unique structure of the eye. Numerous anatomical and physiological constraints such as tear turnover, nasolachrymal drainage, reflex blinking, ocular static and dynamic barriers pose a challenge and impede deeper ocular drug permeation. Corneal epithelium's tight junction acts a selective barrier for entry of small molecules whereas diffusion of macromolecules is prevented by paracellular routes [3]. Hence, less than $5 \%$ of topically applied dose reaches to deeper ocular tissues. Conventional eye-drops exhibit poor corneal permeability, rapid nasolacrimal drainage, low pre-ocular retention time, rapid precorneal clearance, low ocular bioavailability, frequent administration and rapid drainage due to constant tear flow. To overcome these drawbacks lipid based vesicular drug delivery system such as liposomes, transfersomes, transethosomes, niosomes etc are being developed as novel prospective in ocular drug delivery. But with liposomes there are issues related to physical and chemical stability, oxidative-degradation of phospholipids and poor encapsulation of drugs. They are prone to drug leakage and aggregation of vesicles into larger particles. Niosomes and transferosomes have also reported physical stability issues such as drug-leakage and vesicle-fusion. Therefore, nanostructured liquid crystalline particles have been selected as prospective ocular drug-delivery vehicle [4].

Nanostructured liquid crystalline particles also called as cubosomes are discrete, sub-micron cubic nanoparticles consisting of two congruent non-intersecting hydrophilic channels divided by lipid bi-layer. Cubosomes are mainly composed of binary system of glyceryl monooleate (GMO) and water, where monooleate acts as precursor for lipid-bilayer. Surfactant provides electrostatic-barrier among particles to avert contact between them, therefore providing steric stabilization to the formulation. Chemically GMO is mono-easter of glycerol and oleic acid. When assembled into liquid crystalline particles, they mimic to the structure of biological-membrane and allow them to amalgamate with highly lipophilic cornealepithelium. They are assembled in three dimensions as honeycomb (cavernous) structure which is structurally similar to biological membrane that enables these carriers to fuse with lipophilic corneal epithelium for increased topical drug delivery [5]. Honeycomb structure of cubosomes allows them to retain for prolonged duration on corneal membrane contrast to spherical vesicles, providing increased time-period for drug-loaded particles to permeate through cornea. Cubosomes possess high internal surface-area and presents higher encapsulation as they have higher portion of lipid, consequently exhibits large surface area. They can be harnessed to encapsulate diverse molecules of amphiphilic, hydro-phobic and hydro-philic drugs. They possess high thermodynamic stability, bioadhesive nature, biocompatibility, bio-degradability, protect compounds from physical and enzymatic degradation and sustained drug release behaviour [6].

Therefore, with all the above aspects, Vancomycin $\mathrm{HCl}$ loaded cubosomes were developed, optimized and characterized in this investigation. Cubosomes were formulated for prolonged pre-corneal residence time, enhanced targeting, increased permeability, reduced dosing frequency, controlled drug release, reduced 
systemic side-effects, improved bioavailability and high stability. To assess the efficacy of developed cubosomes, ex-vivo corneal permeation studies, in-vivo studies, ocular pharmacokinetic studies and corneal toxicity studies were performed in rabbit's eyes and compared with marketed eye-drops.

\section{Materials And Experimental Methods \\ 2.1. Materials}

Vancomycin $\mathrm{HCl}$ was obtained as gift sample from Cadila Pharmaceuticals Limited, Ahmadabad, India. Glyceryl Monooleate was procured from Gattefose, France. Poloxamer 407 and dialysis membrane was purchased from Sigma Chemical Company (Sigma-Aldrich Corp., St. Louis, MO, USA). All other solvents used were of analytical grade. Double-distilled water was utilized during the study.

\subsection{Methodology}

\subsubsection{Optimization of formulation variables and process variables}

Cubosomes were developed by employing fragmentation method. Distinct concentrations of Glyceryl Monooleate (GMO) ranging from $0.5 \%, 1 \%, 1.5 \%, 2 \%, 2.5 \%, 3 \%, 3.5 \%, 4 \%, 4.5 \%$ and $5 \%$ were used. A constant amount of drug was weighed and disseminate in molten blend of GMO and poloxamer $407(1 \%$ $\mathrm{w} / \mathrm{v}$ ). Similarly, different concentrations of surfactant (poloxamer 407 ) were taken, 0.1 to $1 \%$ and the other parameters were kept constant. Likewise, different stirring time was evaluated to develop the cubosomes so as to optimize the best one. Different time for homogenization was taken as $0.5,1,1.5,2$, $2.5,3,3.5,4,4.5$ and 5 minutes. Rest of the parameters such as concentration of GMO, poloxamer 407 and stirring speed was kept constant. Stirring time was optimized with respect to entrapment efficiency, polydispersity index and particle size of cubosomes. Varying stirring speeds from 1000 to 10000 RPM were optimized so that the formulation doesn't flocculate or aggregate [7].

\subsubsection{Preparation of vancomycin loaded nanostructured liquid crystalline particles}

Cubosomes were developed by virtue of fragmentation of glyceryl monooleate and poloxamer 407 bulk cubic phase gels. Primarily $\mathrm{GMO}$ and poloxamer 407 were entirely liquefied at $60^{\circ} \mathrm{C}$ in a hot water bath, and afterward vancomycin $\mathrm{HCl}$ was incorporated to amalgamate under ceaseless mixing. To accomplish a homogeneous state, water was included moderately and the blend was vortexed. The cubic phase gel was developed following equilibration for 48 hours at room temperature. Subsequently, the cubic gel was distorted by adding nearly $20 \mathrm{ml}$ water during mechanical stirring. Thereupon, high-pressure homogenizer was utilized to homogenize the developed dispersion at high pressures and cycles to form an opalescent dispersion of cubosomes. The final dispersion of nanostructured liquid crystalline particles was stored at room temperature [8]. 


\subsubsection{Taguchi statistical experimental design for optimization of VA-loaded cubosomes}

Taguchi method of design of experiment was employed for the optimization of the formulation, which uses standard orthogonal arrays for forming a matrix of experiments in such a way so as to extract the maximum important information with minimum number of experiments. The fundamental principle of this method serves as screening filters which examine the effects of many process variables and identify those factors which have major effects on process using a few experiments. It included four factors (formulation variables) i.e. lipid concentration (\%v/v), surfactant concentration (\%v/v), stirring time (sec) and stirring speed (rpm). All the factors were assigned three levels i.e. low, medium and high as depicted in Table 1. Based on the number of factors and their levels, $L_{9}\left(3^{4}\right)$ orthogonal array was employed as shown in Table 2. Optimization was done on the basis of 2 techniques i.e. "smaller the better" and "larger the better" and signal to noise ratio ( $\mathrm{S} / \mathrm{N}$ ratio). The signal to noise ratio takes the mean as well as the variation between results. Smaller the better is usually chosen signal to noise ratio for un-desirable characteristics like "defects" when the desired ideal-value is zero, for example size of particles, PDI. Whereas, larger the better is chosen when desired-ideal value is larger, for example entrapment efficiency [9].

Table 1

Experimental control factors and their levels for VA-cubosomes.

\begin{tabular}{|lllll|}
\hline S. No. & Variables & \multicolumn{2}{l|}{ Levels } & \\
\cline { 3 - 5 } & & Low (1) & Medium (2) & High (3) \\
\hline 1 & Concentration of GMO & $1 \%$ & $1.5 \%$ & $2 \%$ \\
\hline 2 & Concentration of Poloxamer 407 & $0.1 \%$ & $0.5 \%$ & $1 \%$ \\
\hline 3 & Stirring Time (mins) & 2 & 2.5 & 3 \\
\hline 4 & Stirring Speed (rpm) & 4000 & 5000 & 6000 \\
\hline
\end{tabular}


Table 2

Formulation table Taguchi $\mathrm{L}_{9}\left(3^{4}\right)$ orthogonal array for

VA-cubosomes.

\begin{tabular}{|lllll|}
\hline Batches & \multicolumn{4}{l}{ Parameters } \\
\cline { 2 - 5 } & A (\%) & B (\%) & C (mins) & D (rpm) \\
\hline F1 & $1(1)$ & $1(0.1)$ & $1(2)$ & $1(4000)$ \\
\hline F2 & $1(1)$ & $2(0.5)$ & $2(2.5)$ & $2(5000)$ \\
\hline F3 & $1(1)$ & $3(1)$ & $3(3)$ & $3(6000)$ \\
\hline F4 & $2(1.5)$ & $1(0.1)$ & $2(2.5)$ & $3(6000)$ \\
\hline F5 & $2(1.5)$ & $2(0.5)$ & $3(3)$ & $1(4000)$ \\
\hline F6 & $2(1.5)$ & $3(1)$ & $1(2)$ & $2(5000)$ \\
\hline F7 & $3(2)$ & $1(0.1)$ & $3(3)$ & $2(5000)$ \\
\hline F8 & $3(2)$ & $2(0.5)$ & $1(2)$ & $3(6000)$ \\
\hline F9 & $3(2)$ & $3(1)$ & $2(2.5)$ & $1(4000)$ \\
\hline
\end{tabular}

2.2.4. Characterization of vancomycin loaded nanostructured liquid crystalline particles

\subsubsection{Drug Excipient Compatibility Studies}

For the ascertainment of compatibility studies of drug and excipients, Fourier Transform-Infrared Spectroscopy (FT-IR) (Perkin Elmer RX1 model) was utilized. Spectra of vancomycin HCl, glyceryl monooleate, poloxamer 407 and mixture of drug and excipients was examined, and then analogized with individual reference spectra. For analysis, $1 \mathrm{mg}$ of test and $\mathrm{KBr}$ was blended in the proportion of $1: 5$ and pellets were developed at high compaction pressure. The spectra were acquired in infrared region of 450$4000 \mathrm{~cm}^{-1}$ at resolution of $4 \mathrm{~cm}^{-1}$ [10].

\subsubsection{Particle Shape and Surface Morphology}

Morphological evaluation of VA-loaded cubosomes was performed utilizing Transmission Electron Microscopy (TEM) (FEI Tecnai TF20). It is a fundamental instrument for legitimately imaging nanoparticles to acquire quantitative analysis of particle size, surface morphology, shape and size distribution. To prepare sample for TEM, a droplet of developed cubosomes was deposited on a 300 mesh copper lattices smeared by amorphous carbon film and then air dried. Surplus fluid was eliminated by an adsorbent paper and cubosomes were scrutinized at 250000X and 10000X magnifications at 200 $\mathrm{kV}$ voltage acceleration [11].

\subsubsection{Particle Size Distribution and Polydispersity Index}


Particle size distribution and polydispersity index (PDI) of cubosomes were analyzed by dynamic light scattering technique utilizing Malvern nano S90 (Malvern Instruments, Worcestershire, UK). Cubosomal formulations were diluted with de-ionized water and poured in cuvette and then placed in analyzing chamber. Temperature was kept at $25^{\circ} \mathrm{C}$ and angle was fixed at $90^{\circ}$ during the analysis [12].

\subsubsection{Zeta Potential Measurement}

Zeta potential is contemplated as crucial indicator for estimation of surface charge and physical stability of nanoparticles. Estimation of zeta potential of cubosomes was ascertained by electrophoretic mobility of particles. In a U-type tube cubosomes were poured in Zetasizer (3000HS Malvern Instruments, Worcestershire, UK) at a temperature of $25^{\circ} \mathrm{C}$ [13].

\subsubsection{Drug Entrapment Efficiency}

Drug entrapment efficiency of VA-loaded cubosomes were determined by calculating the percentage of amount of drug entrapped by cubosomes. The developed formulations were evaluated by centrifugation technique, in this method cubosomes were centrifuged at $12000 \mathrm{rpm}$ for 30 minutes employing refrigerated centrifuge (Remi, C-24BL, New Delhi, India). The resultant suspension was then segregated and supernatant was accumulated. The absorbance of supernatant was analyzed spectrophotometrically at absorption maxima ( $\lambda$ max) $281 \mathrm{~nm}$ by using UV-visible spectrophotometer (UV-1800, Shimadzu, Japan) [14]. The \% EE was calculated by using the formula:

$\% \mathrm{EE}=$ Amount of drug in supernatant $\times 100$

Initial amount of drug added

\subsubsection{In-vitro Drug Release Studies}

The release of vancomycin form cubosomes was demonstrated by employing Franz Diffusion (FD) cell. Dialysis membrane of molecular weight cut off 12000-14000 Da (Spectra Pro, Spectrum Chemical Manufacturing Corp., USA) and pore size was $2.4 \mathrm{~nm}$. Surface area of the dialysis membrane for the release of drug was $3.14 \mathrm{~cm}^{2}$. Receiver compartment was filled with simulated tear fluid (STF) having pH 7.4 as medium. Dialysis membrane was positioned in the FD-cell and in the donor compartment cubosomal preparation was placed [15]. FD-cell was thermostatically maintained at $37^{\circ} \mathrm{C} \pm 1^{\circ} \mathrm{C}$ under constant magnetic stirring. $5 \mathrm{ml}$ of sample were drawn from receiver chamber at pre-determined interludes and concurrently replenished with equivalent quantity of buffer to perpetuate sink conditions. The withdrawn samples were appraised for cumulative percentage drug release by using UV-visible spectrophotometer at $281 \mathrm{~nm}$ and graph was plotted [16].

\subsubsection{Ex-vivo Transcorneal Permeation Studies}

Ex-vivo transcorneal permeation studies were carried out on rabbit cornea utilizing FD-cell. Cornea and 4$5 \mathrm{~mm}$ of scleral tissue was cautiously extracted and rinsed with cold saline to eliminate protein adhered 
to the tissue. Freshly isolated cornea was mounted in-between donor and receptor chamber in such a manner that the epithelial side positioned towards donor chamber and endothelial side towards receptor chamber. Simulated tear fluid of pH 7.4 was added in the receptor chamber and the temperature was thermoregulated at $37^{\circ} \mathrm{C} \pm 1^{\circ} \mathrm{C}$ with persistent magnetic agitation. Aliquot of optimized VA-cubosomal formulation and VA-solution was introduced on the corneal surface for correlative examination. Form the receptor compartment, $1 \mathrm{ml}$ of sample was drawn out at pre-established schedule and afterwards replaced with equivalent volume of STF [17]. The samples were investigated by UV-visible spectrophotometer at $281 \mathrm{~nm}$. The drug permeation \% was determined by following;

Permeation $\%=$ Amount of drug permeated in receptor $\mathrm{x} 100$

\section{An initial amount of drug in donor \\ 2.2.4.8. Corneal Hydration Studies}

The effect of the VA-loaded cubosomes and VA-solution on corneal hydration was determined. In brief, at the end of the experiment, the scleral tissue was removed from cornea; its epithelial surface was wiped with filter paper and weighed (initial weight). The cornea was then soaked in $1 \mathrm{ml}$ of methanol, dried overnight at $90^{\circ} \mathrm{C}$, and reweighed (final weight) [18]. From the difference in weight, corneal hydration (\%) was calculated as follows:

Corneal hydration $(\%)=$ Initial weight - Final weight $/$ Initial weight $\times 100$

\subsubsection{Bioadhesion studies}

Bioadhesion studies were performed to ascertain the potential of developed formulation to cling with corneal surface for protract duration. Rabbit eyes were exercised and cornea was extracted and cleansed. Extricated cornea was kept on petri-dish containing STF with mucosal side of the cornea outwards. Aliquot of formulation was applied to the cornea followed by a droplet of water soluble dye. The ocular tissue was kept hydrated by perpetuating supply of STF. Bioadhesion was visually perceived for its integrity at designated interludes up to 24 hours [19].

\subsubsection{Ocular tolerance test}

Hen's Egg Test/Huhner-Embroynen Test on Chorioallantoic membrane (HET-CAM) is substitute method to draize test done on rabbit eyes. In this investigation ocular irritancy, vascular response, hemorrhage and injuries to conjunctiva are observed for an ophthalmic product. Fresh fertilized hen's egg weighing 50-60 $\mathrm{g}$ were acquired from poultry farm. Briefly, fertilized eggs acquired from poultry farm were incubated for 3 days at $37 \pm 0.5^{\circ} \mathrm{C}$ and $55 \pm 5 \%$ relative humidity [20]. On day 3, albumin (3ml) was drawn-out from egg utilizing sterilized approach and hole was fastened with paraffin sterilized with $70 \%$ alcohol. On day 10 of incubation, at equator $2 \times 2 \mathrm{~cm}$ of egg shell was removed to create a window [21]. Normal saline $(0.9 \%$, negative control), sodium hydroxide $(0.1 \mathrm{~N}$, positive control) and VA-cubosomes (test formulation) were 
administered directly to the surface of CAM. At pre-established interims, CAM was observed for vascular responses/injuries and scores were specified as per scoring scheme. No visible hemorrhage $=0$ (Nonirritant), observable membrane discoloration $=1$ (mild irritant), structures covered slightly because of membrane discoloration/hemorrhage $=2$ (moderately irritant) and structures covered completely owing to membrane discoloration/hemorrhage $=3$ (severe irritant) [22]

\subsubsection{Anti-microbial studies}

Comparative anti-microbial activity of VA-cubosomes and VA-solution were determined utilizing agar welldiffusion technique along with cup-plate technique. There studies were performed on Staphylococcus aureus and Pseudomonas aeruginosa. Media was composed by blending agar and nutrient broth and sterilized by autoclave at $15 \mathrm{lb} / \mathrm{sq}$-inch at $121^{\circ} \mathrm{C}$ for 60 minutes. In two distinct petri-dish, nutrient agar media was poured and was inoculated with $S$. aureus and $P$. aeruginosa in sterile conditions under laminar air flow unit. Subsequently, in the inoculated solidified nutrient-agar plates, two wells of $8 \mathrm{~mm}$ were created with the assistance of sterile cork-borer. The wells were loaded with vancomycin solution as control and developed cubosomes as test and then incubated for 24 hours at $37^{\circ} \mathrm{C}$. The diameter of inhibition zone was calculated and compared with control [23].

\subsubsection{Isotonicity evaluation}

Isotonicity of ophthalmic preparation has to be maintained to avert tissue damage and corneal irritation. Isotonicity assessment was performed by hemolytic method. The developed formulation of VAcubosomes were blended with red blood cells of mice and perceived with inverted microscope at magnification of $45 \mathrm{X}$. The concentration of hypotonic solution was $(0.45 \% \mathrm{w} / \mathrm{v} \mathrm{NaCl})$, hypertonic solution $(3 \% \mathrm{w} / \mathrm{v})$ and isotonic solution $(0.9 \% \mathrm{w} / \mathrm{v} \mathrm{NaCl})$. Aforementioned method was replicated for isotonic solution (negative control), hypotonic solution (positive control) along with hypertonic solution to observe bursting, swelling and cremation effect on red blood cells [24].

\subsubsection{Sterility Studies}

Sterility is a crucial requirement for ophthalmic preparation as contamination may cause ocular infections. Sterility studies are performed to detect the existence of pathogens in the developed ocular formulations [25]. Analysis was executed on the optimized cubosomal formulation by direct inoculation technique as described in Indian Pharmacopoeia 2010. In this study, $2 \mathrm{ml}$ of VA-cubosomes were transferred to two distinct flasks encompassing $10 \mathrm{ml}$ of soybean-casein digest medium and fluid thioglycollate medium. The inoculated media was blended with medium and was incubated at $20^{\circ} \mathrm{C}-25^{\circ} \mathrm{C}$ for soyabean-casein digest media and $30^{\circ} \mathrm{C}-35^{\circ} \mathrm{C}$ for fluid-thioglycollate medium for 14 days. After end of 14 days, the incubated flasks were scrutinized for any indication for growth of microorganisms [26]. 


\subsubsection{Drug Release Kinetics Studies}

For determination of drug release kinetics of developed formulation, various kinetic models were implemented. Data acquired from in-vitro drug release studies was fitted into different kinetic models such as Korsmeyer-Peppas model (log percentage drug release v/s log time), Higuchi's model (percentage of drug release $\mathrm{v} / \mathrm{s}$ square root of time), first order equation (log percentage of drug remaining $\mathrm{v} / \mathrm{s}$ time) and zero order equation (cumulative amount of drug release $\mathrm{v} / \mathrm{s}$ time). For respective models, correlation co-efficient was evaluated [27].

\subsubsection{In-vivo studies}

Rabbits were provided by Central Animal House, Amity University, Noida, India. The study was approved by University Animal Ethical Committee with registration number 1327/PO/ReBi/S/10 /CPCSEA. All institutional and national guidelines for the care and use of laboratory animals were followed. In-vivo studies were performed on rabbit's eyes which were induced with bacterial keratitis. In this investigation, twelve New Zealand albino rabbits weighing 2-3 kg with no sign of ocular inflammation and gross abnormalities were utilized. Rabbits were categorized into 3 groups of 4 rabbits. Group I (control) was instilled with sterile saline solution, group II was topically administered with optimized VA-cubosomes and group III administered with VA-solution. In group III, $100 \mu \mathrm{l}$ of optimized cubosomes were introduced twice a day and in group II vancomycin solution four times a day [28].

S. aureus was grown aerobically in $10 \mathrm{~mL}$ of brain heart infusion broth (HiMedia, India) at $37^{\circ} \mathrm{C}$ for 18 hours. Microorganisms were collected by centrifugation (Remi, C-24BL, New Delhi, India) at $5000 \mathrm{rpm}$ for 10 mins and washed with phosphate buffer saline (PBS) pH 7.4 and suspended in $2 \mathrm{~mL}$ PBS. Keratitis was induced by inoculating $100 \mu \mathrm{L}$. aureus suspension (containing nearly $10^{7}-10^{8}$ colony-forming units) in rabbit's eyes by intrastromal injection with 29-gauge needle. Manifestations of keratitis were developed after 24 hours of microbial inoculation into eyes [29,30]. Rabbit's eyes were evaluated for clinical signs of bacterial keratitis such as redness, mucoid discharge, lacrimal secretion, corneal ulcer and swelling of eyelid. The grading scale was followed on the scale from 0 to 4 as 0 (absent), 1 (mild), 2 (moderate), 3 (severe) and 4 (extensive). The grading of clinical signs were performed at intervals of 0 , 24,72 and 120 hours. The formulations were instilled into eyes of rabbit following the complete development of infection. The eyes were perceived for the recuperation of infected eyes on each successive day by aforementioned categorization till complete recovery from keratitis. Comparative analysis of developed VA-loaded cubosomes with vancomycin solution was done and the treatment results were compared. Significance was determined by applying unpaired t-test [31].

\subsubsection{Ocular pharmacokinetic studies}

The rabbits were randomly divided into the following two groups: a group treated with vancomycin solution and a group treated with VA-cubosomes. Ocular absorption of VA-cubosomes was assessed on 
aqueous humor fluids. Pupils of rabbits were dilated with topical instillation of $0.25 \%$ tropicamide. Rabbits were anesthetized through-out the investigation using injection of sodium pentobarbital (30 $\mathrm{mg} / \mathrm{kg}$ ) injected into marginal ear-vein. The rabbits were administered with $100 \mu \mathrm{l}$ of optimized cubosomal formulation in the lower conjunctival sac of the right eyes and VA-solution in left eyes. Aqueous humor samples $(0.15 \mathrm{ml} \approx 50-80 \mu \mathrm{L})$ were withdrawn at different time intervals at $0.25,0.5,1$, $2,3,4,5$ and 6 hours by anterior chamber paracentesis utilizing $1 \mathrm{ml}$ insulin syringe attached with 26gauge needle. The aqueous humor samples were collected in glass tubes and stored at $-20^{\circ} \mathrm{C}$ [32]. Study was repeated in triplicate and samples were examined utilizing UV-spectrophotometer. The pharmacokinetic parameters such as area under the concentration-time curve $\left(\mathrm{AUC}_{0 \rightarrow t}\right)$ and peak aqueous humor concentration ( $\mathrm{Cmax}$ ) were calculated from appropriate graphs using the linear trapezoidal rule (Kaleidagraph, Synergy Software) [33].

\subsubsection{Corneal Toxicity Studies (Histopathology Studies)}

Histopathology studies were done to corroborate the results obtained from in-vivo studies on developed cubosomes and also to scrutinize corneal structure and integrity. Rabbits were euthanized with injection of sodium pentobarbital and then eyeballs were removed. Rabbit corneas was cautiously exercised and incubated with optimized VA-cubosomes (test sample), sodium dodecyl sulfate $0.1 \% \mathrm{w} / \mathrm{v}$ (positive control) and phosphate buffer saline (negative control) for 2 hours. Following incubation, rabbit corneas were rinsed with phosphate buffer saline ( $\mathrm{pH}$ 6.4). Subsequently, corneas were instantaneously fixed with $8 \% \mathrm{v} / \mathrm{v}$ formalin solution. Corneal tissues were dehydrated with an alcohol gradient and placed in melted paraffin and then hardened to form block. Cross-sections of blocks $<1 \mathrm{~mm}$ were slit and stained with haematoxyline and eosine. The slides were cover-slipped and assessed under microscope [34].

\subsubsection{Stability Studies}

The aim of stability studies was to determine that the developed formulation has preserved its efficacy and productivity with the progression of time, humidity and temperature. Comprehensive stability data was collected for shelf-life determination and slope and degradation constant of VA-loaded cubosomes. The protocols for determination of stability studies were in accord as per ICH guidelines. For evaluation for stability, the developed cubosomal preparation was subjected to distinct humidity and temperature conditions. Optimized VA-cubosomes were kept in screw capped amber colored glass vials and stored at $5 \pm 1^{\circ} \mathrm{C}, 25 \pm 2^{0} \mathrm{C}, 40 \pm 2{ }^{\circ} \mathrm{C}, 60 \pm 2^{0} \mathrm{C}$, and $75 \pm 5 \%$ relative humidity for a period of 6 months. The samples were drawn-out at different interims viz. 0, 30, 60, 90 and 180 days and investigated for entrapment efficiency, particle size distribution, polydispersity index, $\mathrm{pH}$, in-vitro drug release and phase separation [35].

\section{Results And Discussion}




\subsection{Optimization of Formulation Variables and Process Variables}

For optimization of formulation variables and process variables different characterization parameters were considered such as particle size, polydispersity-index and entrapment efficiency. In the optimization of lipid concentration, an increment in particle size and decreased drug entrapment was perceived on increasing the ratio of drug to lipid. On increasing the lipid concentration from $0.5-5 \% \mathrm{v} / \mathrm{v}$, the size of the cubosomes increased from $46.24 \pm 0.58 \mathrm{~nm}$ to $273.55 \pm 0.43 \mathrm{~nm}$. The formulations with concentration of $1 \%, 1.5 \%$ and $2 \%$ glyceryl monooleate demonstrated best appropriateness for ocular delivery. On increasing the concentration of lipid from $0.5 \%-2.5 \% \mathrm{v} / \mathrm{v}$, increased entrapment was observed as large number of cubosomes were formulated which led to higher drug entrapment. Increasing the quantity of glyceryl monooleate may have resulted in faster solidification of cubosomes. But on further augmentation of lipid from $3-5 \% \mathrm{v} / \mathrm{v}$, drug entrapment was decreased which might be due to increase in viscosity that may hinder diffusion of drug in lipid matrix [36].

The concentration of surfactant was optimized so as to acquire cubosomes of small size with highest drug entrapment. $0.1 \%, 0.5 \%$ and $1 \% \mathrm{w} / \mathrm{v}$ concentration of surfactant was considered as optimization concentration as it led to low PDI, low particle size of $46.24 \pm 0.58,82.31 \pm 0.26$ and $68.22 \pm 0.85 \mathrm{~nm}$ with better entrapment efficiency of $87.28 \%, 86.92 \%$ and $89.25 \%$. Poloxamer 407 provided steric stabilization in opposition to accumulation of cubosomes particles by securing its poly-propylene oxide that causes impediment in polar-region or at exterior of glyceryl monooleate bi-layer.

Cubosomes were synthesized at distinct stirring time and 2, 2.5 and 3 minutes was the optimized stirring time. Stirring speed of homogenization was varied from 1000 to $10000 \mathrm{rpm}$. Stirring speed of 4000,5000 and $6000 \mathrm{rpm}$ were optimized as small particle size and high drug entrapment was attained. Higher shear was acquired on escalating the stirring speed that decreased the particle size [37].

\subsection{Drug-Excipient Compatibility Studies}

FT-IR was done to analyze estimate any physico-chemical influence on drug and its excipients and to establish that chosen excipients were compatible with drug. FT-IR spectroscopy of vancomycin $\mathrm{HCl}$, glyceryl monooleate, poloxamer 407 and physical mixture of drug-excipients are depicted in Fig. 1. Pure vancomycin manifested its characteristics sharp peak at $3418.51 \mathrm{~cm}^{-1}$ that was ascribed to $\mathrm{N}-\mathrm{H}$ stretching, $1505.76 \mathrm{~cm}^{-1}$ due to $\mathrm{C}=\mathrm{C}$ stretching, $1383.18 \mathrm{~cm}^{-1}$ indicated $\mathrm{OH}$ phenol group, C-O-C stretching at $1230.26 \mathrm{~cm}^{-1}$ and $1062.47 \mathrm{~cm}^{-1}$ aliphatic amine for $\mathrm{C}-\mathrm{N}$ stretching were in accordance with standard vancomycin FT-IR data [38, 39].

The FT-IR spectrum of pure GMO displayed characteristics peaks in the range of $3423-765 \mathrm{~cm}^{-1}$. Spectra of GMO exhibited broad sharp peak at $3423 \mathrm{~cm}^{-1}$ that could be attributed stretching of $\mathrm{O}-\mathrm{H}$ group, peak at $2926 \mathrm{~cm}^{-1}$ were ascribed to $\mathrm{CH}_{2}$ stretching and the band at $1597 \mathrm{~cm}^{-1}$ indicated presence 
of C-C bonds. Sharp peak at $1384 \mathrm{~cm}^{-1}$ designated nitrate absorption band and peak at $618 \mathrm{~cm}^{-1}$ were mainly due to $\mathrm{C}-\mathrm{H}$ bending [40].

Pure poloxamer 407 exhibited characteristic broad peak at $3425 \mathrm{~cm}^{-1}$ due to $\mathrm{O}-\mathrm{H}$ group stretching, 2889 $\mathrm{cm}^{-1}$ peak indicated $\mathrm{C}-\mathrm{H}$ stretching vibration, sharp peak at $1596 \mathrm{~cm}^{-1}$ corresponded to $\mathrm{C}-\mathrm{C}$ stretching and sharp peak at $1384 \mathrm{~cm}^{-1}$ indicated nitrate absorption band. Peak at $1113 \mathrm{~cm}^{-1}$ corresponded to C-O stretching vibration and slight peak at $766 \mathrm{~cm}^{-1}$ was related to $\mathrm{C}-\mathrm{N}$ stretch of aromatic and peak at 618 $\mathrm{cm}^{-1}$ were due to $\mathrm{C}-\mathrm{H}$ bending $[41,42]$.

Combination of vancomycin $\mathrm{HCl}$ and excipients exhibited absorbance in the range of $3419 \mathrm{~cm}^{-1}$ to 617 $\mathrm{cm}^{-1}$. From the FTIR spectra it was observed that there was compatibility between drug, GMO and poloxamer 407. The combination exhibited similar functional groups with extremely slight shift demonstration outstanding compatibility and no evidence of interaction between physical mixture of drug and excipients [43].

\subsection{Particle Shape and Surface Morphology}

VA-loaded cubosomes were envisaged for particle shape and surface morphology through transmission electron microscopy. TEM photomicrographs illustrated nearly cubic organization with well-dispersed individual particles as shown in Fig. 2. Cubosomes were uniformly distributed without any aggregation that could be accredited to optimum combination of drug, excipient, stirring-time and stirring-speed. Large concentration of surfactant adsorbed on exterior of cubosomes acted as overlaying covering to stabilize cubosomes surface. Whereas low surfactant quantity caused enlargement in particle-size due to expansion in inter-facial pressure that caused accumulation of particles [44, 45].

\subsection{Particle Size Distribution and Polydispersity Index}

Dynamic light scattering (DLS) measurements were employed to assess size distribution and polydispersity index of developed cubosomes. Particles size values were in between $51.11 \pm 0.96 \mathrm{~nm}$ to $158.73 \pm 0.46 \mathrm{~nm}$, whereas PDI was found to be in the range of $0.275 \pm 0.53$ to $0.525 \pm 0.48$. Usually, molecular size should be less than $200 \mathrm{~nm}$ for easy permeation across corneal membrane. The developed formulation had molecular size smaller than $200 \mathrm{~nm}$ that ensures adequate bioavailability, good uniformity and significant potential for effortless permeation. The outcome divulged that particlesize of cubosomes was inversely-proportional to increase in quantity of surfactant [46]. High poloxamer quantity could have reduced surface tension of lipid-phase and aqueous phase that might have reduced particle size. High poloxamer amount might have reduced steric hindrances. On minimizing the amount of surfactant, particle size was expanded because of decreased inter-facial stability that was due to inadequate concentration of poloxamer that caused agglomeration of cubosomes [47]. Particle size distribution and polydispersity index of formulation have been tabulated in Table 3. 
Table 3

Compiled characterization of Vancomycin loaded Cubosomes.

\begin{tabular}{|c|c|c|c|c|c|}
\hline $\begin{array}{l}\text { S. } \\
\text { No. }\end{array}$ & Formulations & $\begin{array}{l}\text { Average particle size } \pm \\
\text { S.D }\end{array}$ & $\begin{array}{l}\text { Zeta Potential } \pm \\
\text { S.D }\end{array}$ & $\begin{array}{l}\text { Polydispersity } \\
\text { Index } \pm \text { S.D }\end{array}$ & $\begin{array}{l}\text { Entrapment } \\
\text { Efficiency } \\
\pm \text { S.D }\end{array}$ \\
\hline 1 & F1 & $158.73 \pm 0.46$ & $-22.85 \pm 0.57$ & $0.467 \pm 0.94$ & $\begin{array}{l}72.181 \pm \\
0.64\end{array}$ \\
\hline 2 & F2 & $112.62 \pm 0.36$ & $-25.04 \pm 0.76$ & $0.513 \pm 0.63$ & $\begin{array}{l}75.535 \pm \\
0.86\end{array}$ \\
\hline 3 & F3 & $98.45 \pm 1.08$ & $-28.16 \pm 0.29$ & $0.281 \pm 0.25$ & $\begin{array}{l}81.322 \pm \\
0.71\end{array}$ \\
\hline 4 & F4 & $92.56 \pm 0.83$ & $-23.63 \pm 0.94$ & $0.352 \pm 0.59$ & $\begin{array}{l}86.437 \pm \\
0.98\end{array}$ \\
\hline 5 & F5 & $76.39 \pm 0.57$ & $-28.74 \pm 0.84$ & $0.488 \pm 0.81$ & $\begin{array}{l}89.693 \pm \\
1.21\end{array}$ \\
\hline 6 & F6 & $71.44 \pm 1.03$ & $-26.22 \pm 0.53$ & $0.337 \pm 0.79$ & $\begin{array}{l}88.178 \pm \\
0.39\end{array}$ \\
\hline 7 & F7 & $68.26 \pm 0.78$ & $-21.53 \pm 0.29$ & $0.292 \pm 0.14$ & $\begin{array}{l}90.711 \pm \\
0.27\end{array}$ \\
\hline 8 & F8 & $51.11 \pm 0.96$ & $-25.94 \pm 0.68$ & $0.275 \pm 0.53$ & $\begin{array}{l}91.624 \pm \\
0.42\end{array}$ \\
\hline 9 & F9 & $86.38 \pm 0.31$ & $-30.36 \pm 0.41$ & $0.525 \pm 0.48$ & $\begin{array}{l}88.512 \pm \\
0.55\end{array}$ \\
\hline
\end{tabular}

\subsection{Zeta Potential Measurement}

Zeta potential measurement was analyzed to ascertain the surface charge of cubosomes that is of great significance for anticipating prolonged stability. Zeta potential revealed that vancomycin cubosomes carried negative charge with mean values of $-21.53 \pm 0.29$ to $-30.36 \pm 0.41$ as represented in Table 3 . $-40 \mathrm{mv}$ to $+40 \mathrm{mv}$ is considered as ideal stability range of zeta potential for formulations. High zeta potential manifests high electric repulsion between dispersed particles which averts agglomeration and provides good stability and dispersion quality [48]. Negative charge could be due to existence of fatty acid in glyceryl monooleate. Non-ionic surfactant might have caused negative charge because of hydroxyl ions anchored on exterior of cubosomes and association of hydroxyl group with aqueous medium. Poloxamer 407 provided good steric stability as it stabilized the formulations by steric hindrances that avoided aggregation [49].

\subsection{Drug Entrapment Efficiency}

Drug entrapment efficiency was evaluated to determine concentration of drug incorporated in VAcubosomes. Entrapment efficiency was found between $72.181 \% \pm 0.64$ to $91.624 \% \pm 0.42$ as shown in 
Table 3. Strong affinity between drug and lipid in cubosomes might have acquired the drug into its inverted-type self assembled nanostructure. Cubic crystalline structure promotes high drug entrapment as they have higher portion of lipid that presents large surface area. Entrapment efficiency was determined by the concentration of glyceryl monooleate and poloxamer [50]. The outcome indicated that with the increase in concentration of lipid and surfactant, the entrapment was also increased. This could be attributed to increased viscosity of the formulation, as the concentration of lipid was increased cubosomal dispersion was solidified faster that could have prevented the diffusion of the drug to external phase medium. On further escalating the concentration of lipid, reduction in entrapment was perceived which could be due to immoderate viscosity of cubosomal dispersion that obstructs dispersion of drug from the binary lipid matrices [51].

\subsection{In-vitro Drug Release Studies}

The release of vancomycin from developed cubosomes was carried-out utilizing FD-cell for 24 hours. The release of drug-loaded formulations ranged from $79.43 \% \pm 0.68$ to $92.32 \% \pm 0.29$ for 24 hours. The invitro release profile of VA-cubosomes manifested that the formulations demonstrated initial burst release up to $14.42 \% \pm 0.54$ and sustained release up to $92.32 \% \pm 0.29$. One probable rational for burst release could be attributed to spreading of free-drug adsorbed onto cubosomes particle surface which could easily diffuse [52]. Whereas, drug encompassed into particle core would release over extended time due to firm entrapment of drug by lipid layer. This pattern of slow release could be ascribed to sedated expulsion of drug from inner-water channels in the cubic phases. Both the properties of initial-burst release and sustained drug release were beneficial in ocular delivery of drug. Initial-burst was favorable for ameliorating permeation of the drug and provided rapid onset drug action, however sustained-release provided action of drug over a protracted time-span. The sustained-release pattern of drug release up to 24 hours would facilitate reduced dosing frequency [53]. In-vitro drug release of vancomycin from cubosomes is depicted in Fig. 3.

\subsection{Ex-vivo Transcorneal Permeation Studies}

Transcorneal permeation studies of VA-cubosomes and VA-solution were evaluated through FD cell on rabbit cornea. On the basis of small particle size, PDI, surface morphology, entrapment efficiency and invitro drug release, formulation F8 was selected as the optimized formulation. The permeation studies demonstrated that a linear-relationship between accumulative permeated vancomycin and time could be perceived. The apparent permeability co-efficient $\left(\mathrm{P}_{\mathrm{app}}\right)$ of VA-loaded cubosomes and vancomycin solution was $4.48 \pm 0.62 \times 10^{6}$ and $1.83 \pm 0.84 \times 10^{6} \mu \mathrm{g} / \mathrm{s} / \mathrm{cm}^{2}$ respectively. Steady state flux (Jss) was found to be $1.45 \pm 0.26 \times 10^{3}$ and $0.67 \pm 0.5910^{3} \mu \mathrm{g} / \mathrm{cm}^{2} / \mathrm{h}$. Compared with vancomycin solution, VAcubosomes demonstrated nearly 2.4 -fold increase in permeability co-efficient and 2.1 -fold increase in steady state flux. Significant higher corneal permeation of optimized formulation could be due to strong mucoadhesive property and nano-dimension of nanostructured liquid crystalline particles. Enhanced permeation of optimized cubosomes via paracellular route could be due to presence of surfactant that could have led to disturbance in tight junction of epithelial cells of cornea. Ameliorated drug permeation could also be due to structural similarity between bi-continuous lipid bi-layer of cubosomes and corneal 
epithelial cells that might permit membrane fusion, facilitating direct drug absorption into corneal cells [54]. Transcorneal permeation profiles of VA-loaded cubosomes and VA-solution are graphically represented in Fig. 4.

\subsection{Corneal Hydration Studies}

The corneal hydration level of normal mammalian cornea is between $75 \%-83 \%$ whereas $83-92 \%$ hydration level denotes damage to cornea. The corneal hydration value of the optimized formulation and vancomycin solution was between 75 to $82 \%$, which indicated that the formulation did not cause any harm to the corneal tissue. Corneal hydration level of VA-cubosomes was $81.68 \% \pm 0.73$ and VA-solution was $79.53 \% \pm 0.29$.

\subsection{Bioadhesion studies}

The purpose of the bioadhesion studies was to perceive the nature of developed cubosomes adherence to cornea tissues to resolve the drainage issue contrast to vancomycin solution. To improve the visibility of the formulation, indicator dye was used. It was observed that the intensity of color diminished progressively over 24 hours time period. This indicated the adherence of cubosomal formulation to corneal surface over the entire duration of study. The optimized formulation F8 remained intact in the corneal surface for 24 hours and that could be attributed to the optimum concentration of lipid and surfactant that prolonged the bioadhesion time.

\subsection{Ocular tolerance test}

HET-CAM assay was employed to assess predictive model for in-vitro irritation by perceiving vascular damage, hemorrhage, hyperemia, coagulation and computing irritation-score. Arteries, veins and capillaries are encompassed in CAM that gives identical reaction as inflammation induced in corneal tissue of rabbits during draize-eye irritancy test. With normal saline, 0 mean score was observed [55]. Optimized VA-cubosomes was subjected as test and showed 0 mean score up to 12 hours and 0 score was found up to 24 hours. Results denoted that formulation was non-irritating in nature and could be well-tolerated and was safe for application to eyes. It could be accredited to bio-compatible and nonimmunogenic nature of cubosomes. Positive control reported hemorrhage and coagulation followed by lysis that confirmed severe irritant nature with mean score of 3 [56]. Figure 5 represents photographs illustrating results of HET-CAM test.

\subsection{Anti-microbial studies}

The anti-microbial efficacy of optimized cubosomal formulation and vancomycin solution was executed against Staphylococcus aureus and Pseudomonas aeruginosa. VA-cubosomes displayed higher growth inhibitory effect as compared to control. The anti-microbial properties exhibited that the diameter of zone of inhibition against $S$. aureus for VA-cubosomal formulation was $36 \mathrm{~mm}$ and against $P$. aeruginosa was 
$30 \mathrm{~mm}$ which was significantly higher than VA-solution. Higher zone of inhibition acquired by synthesized cubosomes indicated that formulation had optimum viscosity that resulted in sustained drug release that maintained minimum inhibitory concentration for extended time-period $[57,58]$. Pictorial presentation of anti-microbial studies is depicted in Fig. 6 .

\subsection{Isotonicity evaluation}

Isotonicity test was performed to examine whether the tone of cell would be influenced by entry of water from the instilled solution as instance of hypo-tonic solution or egress of water as instance of hyper-tonic solution. Hypertonic and hypotonic formulations have a tendency to cause irritation, pain and tissue damage to eyes. Ideally the ophthalmic formulations should be isotonic in nature so that they don't cause discomfort, irritation or swelling. Microscopic observations illustrated that blood cells treated with hypertonic solution seemed shrunk whereas hypotonic solution treated blood cells were swollen. Although, RBCs treated with formulated cubosomes and isotonic solution were unaltered and didn't show lysis or crenation of blood cells. The results implied that VA-cubosomes were isotonic with lachrymal fluid and blood and were found to be non-irritating with no ocular damage [59]. Microscopic images for isotonicity evaluation have been depicted in Fig. 7.

\subsection{Sterility Studies}

For sterility studies, fluid thioglycollate medium was employed to detect aerobic bacteria while soya-bean casein digest was used to detect anaerobic fungi and bacteria. Sterility testing corroborated that there was absence of turbidity and no bacteriostatic and fungistatic activity was perceived after incubation for 14 days. Therefore, the optimized VA-cubosomes were found sterile and free from microorganisms [60].

\subsection{Drug Release Kinetics Studies}

Formulation F8 was optimized on the basis of drug release studies to determine drug release kinetic. Data acquired from in-vitro release profile was fitted into diverse kinetic models. The results manifested that the optimized cubosomal formulation was predominantly elucidated by first order $\left(R^{2}=0.9831, K=\right.$ $0.048)$, followed by higuchi's equation $\left(R^{2}=0.9762, K=19.045\right)$ and zero order $\left(R^{2}=0.8724, K=3.683\right)$. The corresponding plot for korsmeyer-peppas equation indicated good linearity $\left(R^{2}=0.9817\right)$. The release exponent ' $n$ ' was found to be 0.6032 , that indicated non-fickian or anomalous transport. In non-fickian release diffusion, rate of vancomycin release from cubosomes were governed by more than one process i.e. diffusion controlled and swelling controlled release.

\subsection{In-vivo studies}


The optimized VA-cubosomal formulation and VA-solution were subjected to in-vivo studies and treatments effects were compared. Criteria taken for response to drug therapy were redness, lacrimal secretion, mucoid discharge, eye-lid swelling and corneal ulcer. Eyes of rabbits were examined and recorded for ocular damage or abnormal signs to conjunctiva, iris or cornea and systemic side-effects [61]. Scores of cubosomal formulation and VA-solution were compared and results indicated that optimized formulation was efficacious in mitigating manifestation of keratitis as depicted in Table 4. After 120 hours, the score of the redness in group II was $2.927 \pm 0.59$ whereas in group III was considerably lower $0.09 \pm 0.0$. Lacrimal secretion and mucoid discharge in group II was $2.032 \pm 0.63$ and $1.649 \pm 0.19$ whereas in group III it was $0.07 \pm 0.0$ and $0.08 \pm 0.0$ respectively. Intensity of keratitis was reduced after 72 hours by the optimized cubosomes and after 120 hours the symptoms were significantly relived. Images during treatment of infected rabbit eyes at different time intervals are depicted in Fig. 8. Rate of frequency of administration was also lowered as compared to solution. Remarkable better results were acquired with vancomycin cubosomes in reducing the severity of keratitis [62]. Grading of parameters of keratitis was done and the results of un-paired t-test between 2 groups demonstrated that cubosomal formulation showed significantly superior response as shown in Table 5.

Table 4

Grading of parameters of keratitis in group II and group III

\begin{tabular}{|lccll|}
\hline \multicolumn{4}{|l|}{ Grading of parameters of Keratitis (Group II - VA-solution) } \\
\hline Parameters & $\mathbf{0}$ hrs & $\mathbf{2 4} \mathrm{hrs}$ & $\mathbf{7 2} \mathrm{hrs}$ & $120 \mathrm{hrs}$ \\
\hline Redness & $4 \pm 0$ & $3.584 \pm 0.84$ & $2.927 \pm 0.59$ & $1.931 \pm 1.08$ \\
\hline Lacrimal secretion & $3 \pm 0$ & $2.636 \pm 0.51$ & $2.032 \pm 0.63$ & $1.401 \pm 0.36$ \\
\hline Mucoid discharge & $3 \pm 0$ & $2.155 \pm 0.72$ & $1.649 \pm 0.19$ & $1.128 \pm 0.61$ \\
\hline Corneal ulcer & $2 \pm 0$ & $1.673 \pm 0.44$ & $1.035 \pm 0.76$ & $0.729 \pm 0.28$ \\
\hline Swelling of eyelid & $2 \pm 0$ & $1.527 \pm 0.24$ & $1.143 \pm 0.48$ & $0.835 \pm 0.92$ \\
\hline Grading of parameters of Keratitis (Group III - VA-loaded cubosomes) \\
\hline Redness & $4 \pm 0$ & $2.915 \pm 1.05$ & $1.524 \pm 0.26$ & $0.09 \pm 0.0$ \\
\hline Lacrimal secretion & $3 \pm 0$ & $1.562 \pm 0.52$ & $0.841 \pm 0.78$ & $0.07 \pm 0.0$ \\
\hline Mucoid discharge & $3 \pm 0$ & $2.326 \pm 0.41$ & $0.736 \pm 0.37$ & $0.08 \pm 0.0$ \\
\hline Corneal ulcer & $2 \pm 0$ & $0.774 \pm 0.85$ & $0.063 \pm 0.18$ & $0.00 \pm 0.0$ \\
\hline Swelling of eyelid & $2 \pm 0$ & $0.846 \pm 0.73$ & $0.295 \pm 0.14$ & $0.00 \pm 0.0$ \\
\hline
\end{tabular}


Table 5

Significance $t$-test results of group II and group III.

\begin{tabular}{|c|c|c|c|c|}
\hline \multirow[t]{2}{*}{ Parameters } & \multicolumn{4}{|c|}{$\begin{array}{l}\text { Significance } t \text {-test results of VA-solution versus VA-loaded } \\
\text { cubosomes }\end{array}$} \\
\hline & $0 \mathrm{hrs}$ & $24 \mathrm{hrs}$ & $72 \mathrm{hrs}$ & 120 hrs \\
\hline \multirow[t]{2}{*}{ Redness } & $\begin{array}{l}P> \\
0.999\end{array}$ & $P<0.0001$ & $P<0.0001$ & $P=0.002$ \\
\hline & $\begin{array}{l}t=0 \\
\text { (NS) }\end{array}$ & $\mathrm{t}=4.972(\mathrm{ES})$ & $\mathrm{t}=4.451(\mathrm{ES})$ & $\mathrm{t}=4.328(\mathrm{ES})$ \\
\hline \multirow[t]{2}{*}{$\begin{array}{l}\text { Lacrimal } \\
\text { secretion }\end{array}$} & $\begin{array}{l}P> \\
0.999\end{array}$ & $P>0.0471$ & $P>0.0315$ & $P=0.2836$ \\
\hline & $\begin{array}{l}t=0 \\
\text { (NS) }\end{array}$ & $\mathrm{t}=3.562(\mathrm{~S})$ & $\mathrm{t}=2.761(\mathrm{~S})$ & $\mathrm{t}=1.529(\mathrm{NS})$ \\
\hline \multirow[t]{2}{*}{$\begin{array}{l}\text { Mucoid } \\
\text { discharge }\end{array}$} & $\begin{array}{l}P> \\
0.999\end{array}$ & $P=0.0683$ & $P=0.0943$ & $P=0.0001$ \\
\hline & $\begin{array}{l}t=0 \\
\text { (NS) }\end{array}$ & $\mathrm{t}=2.813(\mathrm{~S})$ & $\mathrm{t}=4.252(\mathrm{~S})$ & $\mathrm{t}=8.371(E S)$ \\
\hline \multirow[t]{2}{*}{ Corneal ulcer } & $\begin{array}{l}P> \\
0.999\end{array}$ & $P=0.0527$ & $P=0.0753$ & $P=0.0284$ \\
\hline & $\begin{array}{l}t=0 \\
\text { (NS) }\end{array}$ & $\mathrm{t}=3.426(\mathrm{MS})$ & $t=2.526(M S)$ & $t=2.627(S)$ \\
\hline \multirow[t]{2}{*}{ Swelling of eyelid } & $\begin{array}{l}P> \\
0.999\end{array}$ & $P=0.0581$ & $P=0.0923$ & $P=0.9381$ \\
\hline & $\begin{array}{l}t=0 \\
\text { (NS) }\end{array}$ & $\mathrm{t}=1.803(\mathrm{~S})$ & $t=1.694(S)$ & $\mathrm{t}=0.4102(\mathrm{NS})$ \\
\hline
\end{tabular}

\subsection{Ocular pharmacokinetic studies}

Optimized cubosomal formulation and VA-solution were subjected to in-vivo ocular pharmacokinetic studies to evaluate level of drug in aqueous humor. Conventional ocular formulations have number of problems associated with them such as pre-corneal drug loss and poor bioavailability. For a successful therapy it is important to maintain drug in aqueous humor to enhance bio-availability of drug. The results depicted that drug concentration in aqueous humor showed a maximum at 1 hour after administration. Higher concentration of antibiotic is always preferable at early time of infection. The area under curve (AUC) value of cubosomal formulation was $307.63 \pm 0.43 \mu \mathrm{g} \mathrm{min} / \mathrm{ml}$ and VA-solution was $137.72 \pm 0.21$ $\mu \mathrm{g} \mathrm{min} / \mathrm{ml}$, which was 2.1 -fold higher. Whereas, the peak concentration $\left(\mathrm{C}_{\max }\right)$ of VA-cubosomes was $3.16 \pm 0.27 \mu \mathrm{g} / \mathrm{ml}$ whereas VA-solution was $1.24 \pm 0.59 \mu \mathrm{g} / \mathrm{ml}$ which was nearly 2.5 -fold higher $(p<0.01)$. 
The time to peak concentration ( $T_{\max }$ ) of cubosomal formulation was about 1.9-fold higher and mean residence time was 2.2 -fold greater than VA-solution [63]. The transcorneal penetration of vancomycin might be increased due to interaction between cubosomes and cornea in presence of glyceryl monooleate. Moreover, prolonged mean residence time and $\mathrm{T}_{\max }$ could be attributed to close adherence of cubosomes to ciliary muscle and conjunctival sac that would provide subsequent absorption and surface-lipid exchange effect [64]. That might form drug depot at corneal area and would provide more drug to anterior chamber of eyes. Increased ocular-bioavailability could be ascribed to 3 factors: enhanced drug-loading capacity, permeation capability of drug through corneal membrane and ocular contact-time of cubosomes. These factors corroborated intimate contiguity in-between drug and epithelial mucosa, averting tear-washout and therefore correlated with sustained-release of drug and protracted drug-retention [65]. Concentration-time profiles of tobramycin in aqueous humor is graphically represented in Fig. 9, whereas pharmacokinetic parameters are depicted in Table 6.

Table 6

Pharmacokinetic parameters of VA-solution and VA-cubosomes in aqueous humor after topical administration.

\begin{tabular}{|c|c|c|}
\hline Parameters & VA-solution & VA-cubosomes \\
\hline $\mathrm{AUC}_{(0-360)}(\mu \mathrm{g} \mathrm{min} / \mathrm{ml})$ & $137.72 \pm 0.21$ & $307.63 \pm 0.43$ \\
\hline$C_{\max }(\mu \mathrm{g} / \mathrm{ml})$ & $1.24 \pm 0.59$ & $3.16 \pm 0.27$ \\
\hline $\mathrm{T}_{\max }(\min )$ & $30.65 \pm 0.18$ & $58.55 \pm 0.38$ \\
\hline Mean Residence Time (min) & $49.26 \pm 0.46$ & $107.87 \pm 0.92$ \\
\hline
\end{tabular}

Histopathological studies confirmed the results obtained from in-vivo studies. The cross-section of ocular tissues of rabbit were treated with optimized VA-cubosomes, sodium dodecyl sulfate and phosphate buffer saline as illustrated in Fig. 10. Histopathological studies were performed to corroborate the existence of normal corneal tissues by retaining their morphology without causing impairment to tissues. In positive control, epithelial layer was grievously damaged with absolute separation from bowman's layer at diverse regions of corneal epithelium. Distorted cells were evidently observable with significant corneal swelling. Whereas, corneal tissues incubated with normal saline and VA-cubosomes demonstrated no cytotoxicity and intact epithelial layer with no changes in corneal cross-sections. The corneal layers displayed well-maintained morphology without any stromal swelling, consequently indicating safety of formulation [66].

\subsection{Stability studies}


Stability studies were performed over a period of 6 months and formulations were stored at varied temperatures $5 \pm 1^{0} \mathrm{C}, 25 \pm 22^{\circ} \mathrm{C}, 40 \pm 2^{0} \mathrm{C}, 60 \pm 2^{\circ} \mathrm{C}$, and $75 \pm 5 \% \mathrm{RH}$ to analyze the stability. The optimized cubosomes were observed for entrapment efficiency, particle size distribution, polydispersity index, $\mathrm{pH}$, invitro drug release, precipitation and phase separation. The formulation exhibited satisfactory results and remained stable during 6 months. There were no changes in the physical appearance and no precipitation and phase separation was observed. Slight changes were perceived in the formulation at $60^{\circ} \mathrm{C}$. Table 7 illustrates slope and degradation constant.

Table 7

Slope and Degradation constant of VA-cubosomes.

\begin{tabular}{|llllll|}
\hline Temperature & Slope & Degradation Constant, K & Log K & $1 / \mathrm{T} \times 10^{-3}$ & Log K+4 \\
\hline $\mathbf{5}^{\circ} \mathbf{C}$ & $2.58 \times 10^{-5}$ & $0.538 \times 10^{-4}$ & -4.3825 & 3.529738 & 0.1251 \\
\hline $\mathbf{2 5 ^ { \circ } \mathbf { C }}$ & $3.49 \times 10^{-5}$ & $0.764 \times 10^{-4}$ & -4.9593 & 3.203622 & 0.1843 \\
\hline $\mathbf{4 0 ^ { \circ } \mathbf { C }}$ & $4.21 \times 10^{-5}$ & $1.516 \times 10^{-4}$ & -3.6421 & 3.174781 & 0.1905 \\
\hline $\mathbf{6 0 ^ { \circ } \mathbf { C }}$ & $7.73 \times 10^{-5}$ & $1.924 \times 10^{-4}$ & -3.3158 & 3.091563 & 0.2182 \\
\hline
\end{tabular}

\subsection{Shelf Life Determination (Arrhenius plot)}

Shelf-life determination was calculated by Arrhenius plot. Value of log K was obtained -4.2485 by extrapolation of Arrhenius plot. Optimized VA-cubosomes exhibited shelf-life in range of 3.5 years. Consequently, an arbitrary shelf-life of 3 years could be assigned to cubosomal formulation.

\section{Conclusion}

In the present study, novel nanostructured liquid crystalline particles were developed using glyceryl monooleate and poloxamer 407 by fragmentation method. The prospect of cubosomes as carrier for topical administration was established by the results acquired, demonstrating drug penetration. FTIR results revealed that there was no interaction between the drug and excipient of the formulations. TEM confirmed cubic organization with well-dispersed individual particles. HET-CAM assay denoted that the formulation was non-irritating in nature and could be well-tolerated and was safe for application to eyes. Ex-vivo studies revealed significantly high corneal permeation of optimized formulation. Whereas, in-vivo studies demonstrated that the intensity of keratitis was reduced after 72 hours and after 120 hours the symptoms were significantly relived. Ocular pharmacokinetic studies evaluated drug level in aqueous humor and results revealed that AUC, $\mathrm{T}_{\max }, \mathrm{C}_{\max }$ and MRT of cubosomal formulation was considerably higher than vancomycin solution. Histological evaluation revealed that VA loaded cubosomes demonstrated no cytotoxicity and corneal layers displayed intact epithelial layer without any deleterious 
influence on corneal structure or stromal swelling, consequently indicating safety of formulation. It could be concluded that the developed VA-loaded cubosomes have great potential as viable substitute as compared to conventional eye-drops.

\section{Declarations}

\section{Ethics approval and consent to participate}

The study was reviewed and approved by the Institutional Animal Ethical Committee with registration number 1327/PO/ReBi/S/10 /CPCSEA. All institutional and national guidelines for the care and use of laboratory animals were followed.

\section{Consent for publication}

All authors agree to the submission of this research article to the journal.

\section{Availability of data and materials}

All data generated and analysed during this study are included in this article.

\section{Competing interests}

The authors declare no conflict of interest, financial or otherwise.

\section{Funding}

Not Applicable.

\section{Authors' contributions}

Shreya Kaul: Methodology, Investigation, Writing - original draft. Upendra Nagaich and Navneet Verma: Conceptualization, Supervision, Writing - review \& editing.

\section{Acknowledgements}

None

\section{References}

1. Anutra K, Somsanguan A, Phuriwat L, Chutiporn L, Robert M. Chitosan as an ocular drug delivery vehicle for vancomycin. J Appl Polym Sci. 2010;122:3160-3167. https://doi.org/10.1002/app.34323

2. Zavarshani M, Ahmadi M, Saei HD, Tehrani AA, Naghadeh BD. Comparison therapeutic effects of ciprofloxacin, silver nanoparticles and their combination in the treatment of Pseudomonas keratitis in rabbit: an experimental study. Iran J Pharm Res. 2019;18(1);320-327. 22037/IJPR.2019.2328 
3. Pooja P, Roma M, Sreeja MK. Development and optimisation of quercetin cubosomes incorporated in glyceryl monooleate aided by design expert software. Human. 2018;11(4):80-106. https://www.ijppr.humanjournals.com/wpcontent/uploads/2018/04/8.Pooja-Poulose-RomaMathew-Sreeja-M.K.pdf

4. Swati C, Neha G, Upendra N. Fabrication and evaluation of ultra deformable vesicles for atopic dermatitis as topical delivery. Int J Polym Mater Polym Bioma. 2018;68(5):266-277. https://doi.org/10.1080/00914037.2018.1443932

5. Nilesh RR, Suprit DS, Nishikant AR, Jayashree BT, Pramod BK, Vivek SD. Nanostructured cubosomes in a thermoresponsive depot system: an alternative approach for the controlled delivery of docetaxel. AAPS PharmSciTech. 2016;17(2):436-445. https://doi.org/10.1208/s12249-015-0369-y

6. Shreya K, Neha J, Jaya P, Upendra N. Investigating the retention potential of chitosan nanoparticulate gel: design, development, in-vitro \& ex-vivo characterization. Recent Pat Antiinfect Drug Discov. 2020;15(1):41-67. DOI: 2174/1574891x14666191014141558

7. Yuanfeng W, Jianjun Z, Yazhen Z, Yaxiang G, Meng F, Chengran L, Liang X, Changquan CS, Yuan $G$, Shuai, Q. Cubosomes with surface cross-linked chitosan exhibit sustained release and bioavailability enhancement for vinpocetine. RSC Adv. 2019;9:62876298.https://doi.org/10.1039/C8RA10302J

8. Spicer PT, Hayden KL, Lynch ML, Ofori-Boateng A, Burns JL. Novel process for producing cubic liquid crystalline nanoparticles (cubosomes). Langmuir. 2001;17(19): 5748-56. http://pubs.acs.org/doi/abs/10.1021/la010161w

9. Gulati N, Nagaich U, Saraf SA. Intranasal delivery of chitosan nanoparticles for migraine therapy. Sci Pharm. 2013;81(3):843-854. Doi: 3797/scipharm.1208-18

10. Faiza F, Ayesha N, Shakil A, Syed THS, Muhammad IB. A green approach for the determination of selected anti-diabetic drugs in pharmaceutical formulation by transmission FTIR spectroscopy. $\mathrm{J}$ Braz Chem Soc. 2014;25(11):2033-2038. https://doi.org/10.5935/0103-5053.20140188

11. Nasra M, Ghoraba MK, Abdelazem A. In vitro and in vivo evaluation of cubosomes containing 5fluorouracil for liver targeting. Acta Pharmaceutica Sinica B. 2015;5(1):79-88. DOI: 1016/j.apsb.2014.12.001

12. Bei D, Zhang T, Murowchick JB, Youan BB. Formulation of dacarbazine-loaded cubosomes. Part III. Physicochemical characterization. AAPS PharmSciTech. 2010;1(3): 1243-1249. doi: 1208/s12249010-9496-7

13. Boyd BJ. Characterization of drug release from cubosomes using the pressure ultrafiltration method. Int J Pharm. 2003;260(2):239-247. DOI: 1016/s0378-5173(03)00262-x

14. Rizwan SB, Hanley T, Boyd BJ, Rades T, Hook S. Liquid crystalline systems of phytantriol and glyceryl monooleate containing a hydrophilic protein: characterisation, swelling and release kinetics. J Pharm Sci. 2009;98(11):4191-4204. DOI: 1002/jps.21724

15. Verma D, Kaul S, Jain N, Nagaich U. Fabrication and characterization of ocular phase transition systems for blepharitis: A novel approach. Drug Delivery Letters. 2020;10(1): 24-37. 


\section{DOI: $2174 / 2210303109666190614115304$}

16. Meetali M, Pravin K. Preparation and in vitro/ex vivo evaluation of moxifloxacin-loaded plga nanosuspensions for ophthalmic application. Sci Pharm. 2013;81(2):591-606. doi: 3797/scipharm.1204-16

17. Neeraj M, Gurpreet K. Investigations on polymeric nanoparticles for ocular delivery. Advances in Polymer Technology. 2019;3:1-14. https://doi.org/10.1155/2019/1316249

18. Waghulde V, Saudagar R. Formulation development and evaluation of $\mathrm{pH}$ triggered in-situ ophthalmic gel of besifloxacin hydrochloride. JDDT. 2018;8(5):313-321.

DOI https://doi.org/10.22270/jddt.v8i5.1874

19. Meraj A, Jovita K, Poonam P, Malti A, Alok Y, Shubhini S. Evaluation of a polymer-lipid-polymer system utilising hybrid nanoparticles of dapsone as a novel anti acne agent. Curr Drug Ther. 2016;11(2):86-100. DOI: 2174/1574885511666160818145920

20. Maria CPPRM, Suzana GL, Cristal CC, Alane BV, Ronald SS, Octávio AFP, Álvaro ACL. Gilda GL, Eduardo RJ, Elisabete PS. In-vitro and in-vivo evaluation of efficacy and safety of photoprotective formulations containing antioxidant extracts. Brazilian Journal of Pharmacognosy. 2016;26(2):251258. https://doi.org/10.1016/j.bjp.2015.11.006

21. Narjes S,Saeide S, Hashem KB, Esmael S. In vitro antibacterial effects of silver nanoparticles synthesized using Verbena officinalis leaf extract on Yersinia ruckeri, Vibrio cholera and Listeria monocytogenes. Iran J Microbiol. 2018;10(6):400-408. https://www.ncbi.nlm.nih.gov/pmc/articles/PMC6414745/

22. Kumar D, Jain N, Gulati N, Nagaich U. Nanoparticles laden in situ gelling system for ocular drug targeting. J Adv Pharm Technol Res. 2013;4(1):9-17. DOI: 4103/2231-4040.107495

23. Puranik KM, Tagalpallewar AA. Voriconazole in situ gel for ocular drug delivery. SOJ Pharm Pharm Sci. 2015;2(2):1-10. DOI: http://dx.doi.org/10.15226/2374-6866/2/2/00128

24. Gupta H, Aqil M, Khar RK, Ali A, Bhatnagar A, Mittal G. Biodegradable levofloxacin nanoparticles for sustained ocular drug delivery. J Drug Targ. 2011;19(6): 409-417. DOI: 3109/1061186X.2010.504268

25. Khames A, Khaleel MA, El-Badawy MF, El-Nezhawy. Natamycin solid lipid nanoparticles - sustained ocular delivery system of higher corneal penetration against deep fungal keratitis: preparation and optimization. Int J Nanomedicine. 2019;14:2515-2531. DOI: 10.2147/IJN.S190502

26. Snehal SC, Jameel ASM. A novel corticosteroid cubosomes - for ocular drug delivery. Indo Am J Pharm Res. 2020;10(6):775-784. https://doi.org/10.5281/zenodo.3923714

27. Neupane YR, Srivastava M, Ahmad N. Lipid based nanocarriers system for the potential oral delivery of decitabine: formulation design, characterization, ex vivo, and in vivo Int J Pharm. 2014;477(1):601-612. DOI: 10.1016/j.ijpharm.2014.11.001

28. Soni K, Mujtaba A, Kohli K. Lipid drug conjugate nanoparticle as a potential nanocarrier for the oral delivery of pemetrexed diacid: formulation design, characterization, ex vivo, and in vivo Int J Biol Macromol. 2017;103:139-51. DOI: 10.1016/j.ijbiomac.2017.05.015 
29. Prabhu P, Dubey A, Parth V, Ghate V. Investigation of hydrogel membranes containing combination of gentamicin and dexamethasone for ocular delivery. Int J Pharma Investig. 2015;5(4):214-225.

Doi: 4103/2230-973X.167684

30. Guohua W, Qixia N, Chen Z, Baoxian Z, Qiong Z, Gan L, Shuang W. Self-assembled thermoresponsive nanogels prepared by reverse micelle / positive micelle method for ophthalmic delivery of muscone, a poorly water-soluble drug. J Pharm Sci. 2016;105(9): 2752-2759. DOI: 1016/j.xphs.2016.02.014

31. Aggarwal D, Pal D, Mitra AK, Kaur IP. Study of the extent of ocular absorption of acetazolamide from a developed niosomal formulation, by microdialysis sampling of aqueous humor. Int J Pharm. 2007;338(1-2):21-26. DOI: 1016/j.jpharm.2007.01.019

32. Shun H, Jin-qiu S, Yong G, Hai-ming G, Xin-xin Z, Chun-liu Z, Li G. Novel vehicle based on cubosomes for ophthalmic delivery of flurbiprofen with low irritancy and high bioavailability. Acta Pharmacologica Sinica. 2010;31(8):990-998. DOI: 10.1038/aps.2010.98

33. Jin X, Zhang ZH, Li SL, Sun E, Tan XB, Song J, Jia XB. A nanostructured liquid crystalline formulation of 20(S)-protopanaxadiol with improved oral absorption. Fitoterapia. 2013;84: 64-71. DOI: 1016/j.fitote.2012.09.013

34. Bei D, Marszalek J, Youan BB. Formulation of dacarbazine-loaded Cubosomes--part II: influence of process parameters. AAPS PharmSciTech. 2009;10(3):1040-1047. DOI: 10.1208/s12249-009-9296-0

35. Siekmann B, Bunjes H, Koch MH, Westesen K. Preparation and structural investigations of colloidal dispersions prepared from cubic monoglyceride-water phases. Int J Pharm. 2002;244(1-2):33-43. DOI: 1016/s0378-5173(02)00298-3

36. Kesavan K, Nath G, Pandit J. Preparation and in vitro antibacterial evaluation of gatifloxacin mucoadhesive gellan system. Daru. 2010;18(4):237-

246. https://www.ncbi.nlm.nih.gov/pmc/articles/PMC3304351/

37. Kaul S, Gulati N, Verma D, Mukherjee S, Nagaich U. Role of Nanotechnology in Cosmeceuticals: A Review of Recent Advances. Journal of Pharmaceutics. 2018. https://doi.org/10.1155/2018/3420204

38. Asep BDN, Rosi O, Risti R. How to read and interpret FTIR spectroscope of organic material. J Sci Technol. 2019;4(1):97-118. https://doi.org/10.17509/ijost.v4i1.15806

39. Sayeta G, Sinegre M, Reguiga MB. Development of a Fourier transform infrared spectroscopy coupled to UV-Visible analysis technique for aminosides and glycopeptides quantitation in antibiotic locks. Annales Pharmaceutiques Françaises. 2014;72(1):41-50. DOI: 1016/j.pharma.2013.10.002

40. Venkatesh B, Indira S, Srinivas P. Formulation and evaluation of miconazole nitrate as a cubosomal topical gel. 2014;5(4):2037-2047. http://www.jgtps.com/admin/uploads/QP7hd3.pdf

41. Worle G, Siekmann B, Koch MHJ, Bunjes H. Transformation of vesicular into cubic nanoparticles by autoclaving of aqueous monoolein/poloxamer dispersions. Eur J Pharm. Sci. 2006;27(1):44-53. DOI: 1016/j.ejps.2005.08.004

42. Dawoud MZ, Nasr M. Comparison of drug release from liquid crystalline monoolein dispersions and solid lipid nanoparticles using a flow cytometric technique. Acta Pharmaceutica Sinica B. 
2016;6(2):163-169. https://doi.org/10.1016/j.apsb.2016.01.004

43. Esposito E, Cortesi R, Drechsler M, Paccamiccio L. Cubosome dispersions as delivery systems for percutaneous administration of indomethacin. Pharm Res. 2005;22(12):2163-2173.

DOI: 1007/s11095-005-8176-x

44. Seyedeh PA, Iris RR, Ben JB, Watson L. Impact of preparation method and variables on the internal structure,morphology, and presence of liposomes in phytantriol-Pluronic $B F 127$ cubosomes. Colloids and Surfaces B: Biointerfaces. 2016;145:845-853. DOI: 10.1016/j.colsurfb.2016.05.091

45. Kojarunchitt T, Hook S, Rizwan S, Rades T, Baldursdottir S. Development and characterisation of modified poloxamer 407 thermoresponsive depotsystems containing cubosomes. Int J Pharm. 2011;408(1-2):20-26. DOI: 1016/j.ijpharm.2011.01.037

46. Honary S, Zahir F. Effect of zeta potential on the properties of nano-drug delivery systems-A review (part 2). J. Pharm. Res. 2013;12(2):265-273. DOI: 10.4314/tjpr.v12i2.20

47. Salwa S, Azza AM, Amany OK. Etodolac transdermal cubosomes for the treatment of rheumatoid arthritis: ex vivo permeation and in vivo pharmacokinetic studies. Drug Deliv. 2017;24(1):846-856. DOI: 1080/10717544.2017.1326539

48. Xin P,Ke H, Xinsheng P, Zhiwen Y, Lingzhen Q, Chune Z, Xintian H, Xuan S, Linghui D, Ming L, Chuanbin W. Nanostructured cubosomes as advanced drug delivery system. Curr Pharm Des. 2013;19(35):6290-6297. DOI: 2174/1381612811319350006

49. Jian-Chun L, Na Z, Jin-Xiu Z, Wen-Jing Z, Hong-Min Z, Qing-Qing W, Xiao-Xiang W, Xiu W, Jin Z, Ji-Fu $\mathrm{H}$. Self-assembled cubic liquid crystalline nanoparticles for transdermal delivery of paeonol. Med Sci Monit. 2015;21:3298-3310. DOI: 12659/msm.894484

50. Sadhu VR, Beram NS, Kantamneni P. A review on cubosome: The novel drug delivery system. GSC Biological Pharm Sci. 2018;5(1):76-81. 30574/gscbps.2018.5.1.0089

51. Larsson K. Cubic lipid-water phases: structures and biomembrane aspects. J Phys Chem. 1989;93(21):7304-14. https://doi.org/10.1021/j100358a010

52. Zheng DD, Dai WT, Zhang DR, Duan CX, Jia LJ, Liu Y. In vivo studies on the oridonin-loaded nanostructured lipid carriers. Drug Deliv. 2012;19(6):286-291. DOI: 10.3109/10717544.2012.704096

53. Achouri D, Sergent M, Tonetto A, Piccerelle P, Andrieu V, Hornebecq V. Self-assembled liquid crystalline nanoparticles as an ophthalmic drug delivery system. Part II: optimization of formulation variables using experimental design. Drug Dev Ind Pharm. 2015;41(3): 493-501.

DOI: 3109/03639045.2014.884113

54. Abhishek KS, Preeti KS, Vinod KV. PLGA nanoparticles for ocular delivery of loteprednol etabonate: a corneal penetration study. Artifical cells. Nanomedicine and Biotechnology, 2017;45(6):1156-1164. https://doi.org/10.1080/21691401.2016.1203794

55. Aminu I,Maznah I, Mustapha UI, Rozi M, Ismaila MS, Zuki ABZ. Toxicity Evaluation, HET-CAM Irritation, and Anti-Irritant Potential of Rice Bran Wax Policosanol Nanoemulsion. J Nano Res. 2017;49:44-55. https://doi.org/10.4028/www.scientific.net/JNanoR.49.44 
56. Spielmann, H. HET-CAM test. Methods in Molecular Biology. 1995;43:199-204. DOI: 10.1385/089603-282-5:199

57. Nazoori ES, Kariminik, A. In vitro evaluation of antibacterial properties of zinc oxide nanoparticles on pathogenic prokaryotes. J Appl Biotechnol Rep. 2018;5(4):162-165.10.29252/JABR.05.04.05

58. Young-Ki J, Byung HK, Geunhwa J. Antifungal Activity of Silver lons and Nanoparticles on Phytopathogenic Fungi. Plant Disease. 2009;93(10):1037-1043. https://doi.org/10.1094/PDIS-93-101037

59. Ankit A, Prakash G, Renukaradhya C, Basavaraj MD. Preparation of gellan gum and chitosan based in-situ gel of timolol maleate for ophthalmic drug delivery and evaluation of physicochemical properties and drug release profile. Acta Scientific Pharm Sci. 2019; 3(2):68-78. https://www.actascientific.com/ASPS/pdf/ASPS-03-0205.pdf

60. Shanmugam S, Valarmathi S, Satheesh K. Sterility testing procedure of ophthalmic ocusert aciclovir used for treating herpes simplex virus. Asian J Pharm Clin Res. 2017;10(10):344-346. https://doi.org/10.22159/ajpcr.2017.v10i10.19216

61. Evren HG, Giuseppina S, Sait E, Bonferoni, MC, Güneri T, Caramella C. Cyclosporine A-loaded solid lipid nanoparticles:ocular tolerance and in vivo drug release in rabbit eyes. Curr Eye Res. 2009;34(11):996-1003. DOI: 3109/02713680903261405

62. Jialu W, Fang Z, Rui L, Jingjing C, Qinghua Z, Ruijuan L, Ze W, Xin J, Changxiao Liu. Novel cationic lipid nanoparticles as an ophthalmic delivery system for multicomponent drugs: development, characterization, in vitro permeation, in vivo pharmacokinetic, and molecular dynamics studies. Inter J Nanomed. 2017;12:8115- 8127. DOI: 10.2147/IJN.S139436

63. Anand BS, Atluri H, Mitra AK. Validation of an ocular microdialysis technique in rabbits with permanently implanted vitreous probes: systemic and intravitreal pharmacokinetics of fluorescein. Int J Pharm. 2004;281(1-2):79-88. DOI: 1016/j.ijpharm.2004.05.028

64. Macha S, Mitra AK. Ocular pharmacokinetics in rabbits using a novel dual probe microdialysis technique. Exp Eye Res. 2001;72(3):289-299.1006/exer.2000.0953

65. Rui L, Shuangshuang W, Shiming F, Jialu W, Jingjing C, Xingguo H, Xin H, Changxiao L. Liquid crystalline nanoparticles as an ophthalmic delivery system for tetrandrine: development, characterization, and in vitro and in vivo evaluation. Nanoscale Research Letters. 2016;11:254-266. Doi: 1186/s11671-016-1471-0

66. Lyla PA, Seevakan K. Advancement of Cubosomes Nanomedicine. International Journal of Pure and Applied Mathematics. 2018;119(12):7847-7854. https://acadpubl.eu/hub/2018-11912/articles/3/683.pdf

\section{Figures}




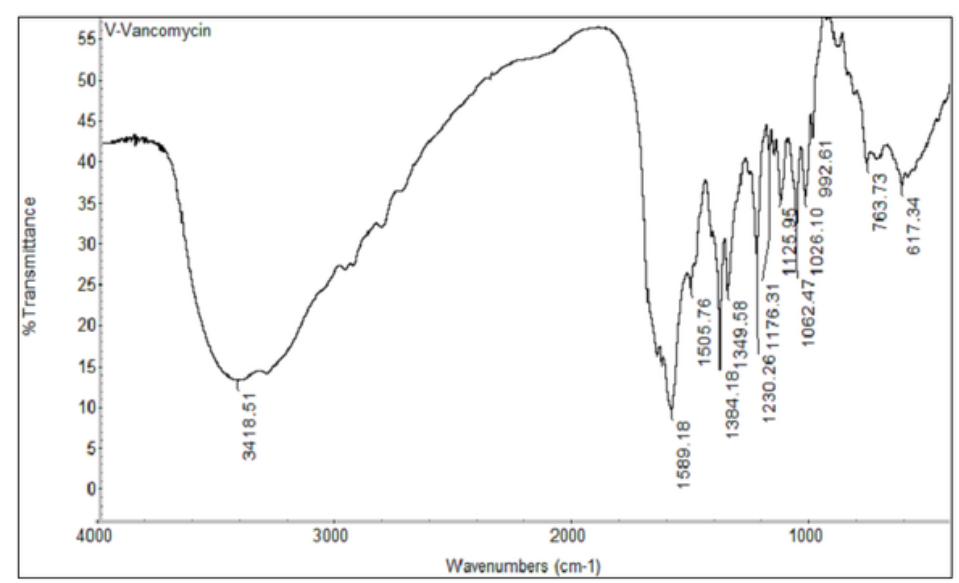

(a)

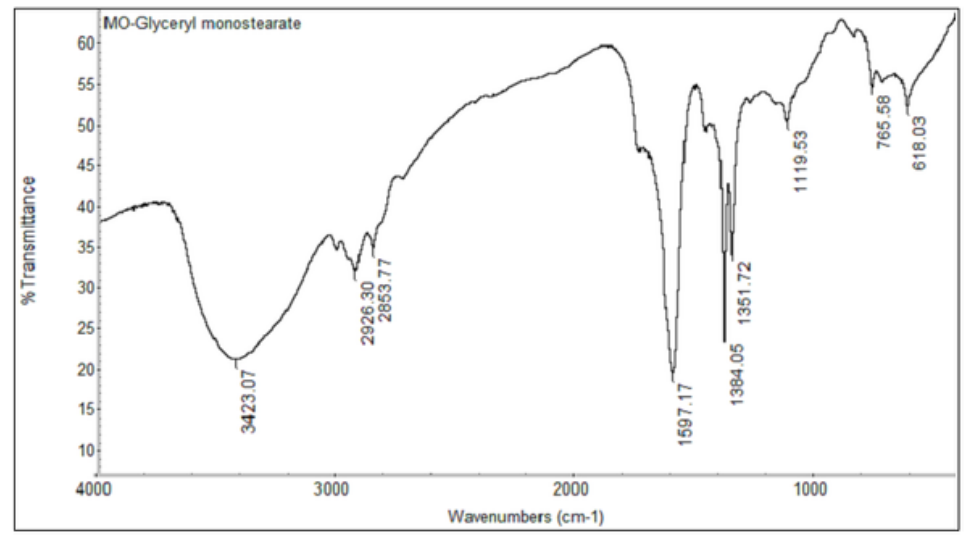

(b)

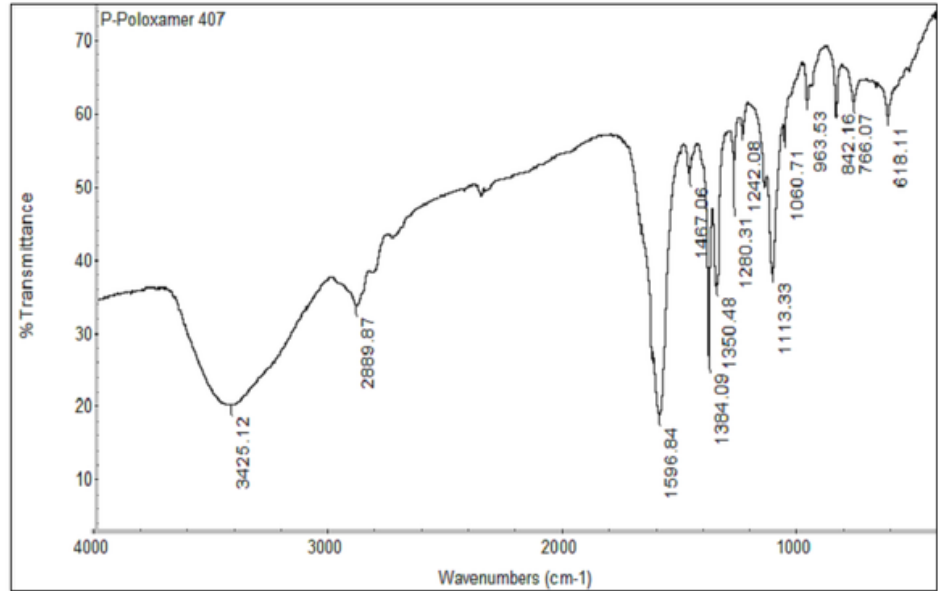

(c)

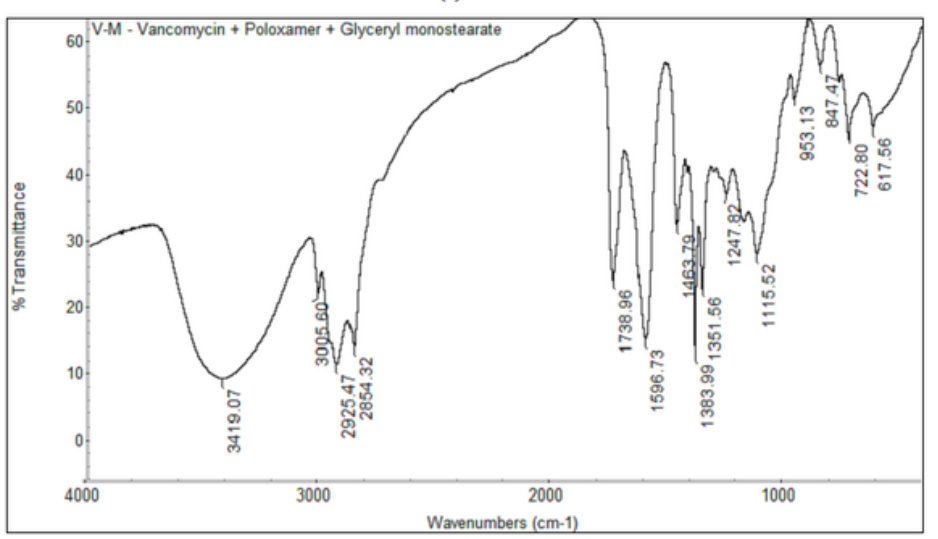

(d)

\section{Figure 1}

FT-IR spectra of (a) Vancomycin HCl, (b) Glyceryl monooleate, (c) Poloxamer 407 and (d) Physical mixture of Vancomycin $\mathrm{HCl}$ and excipients. 

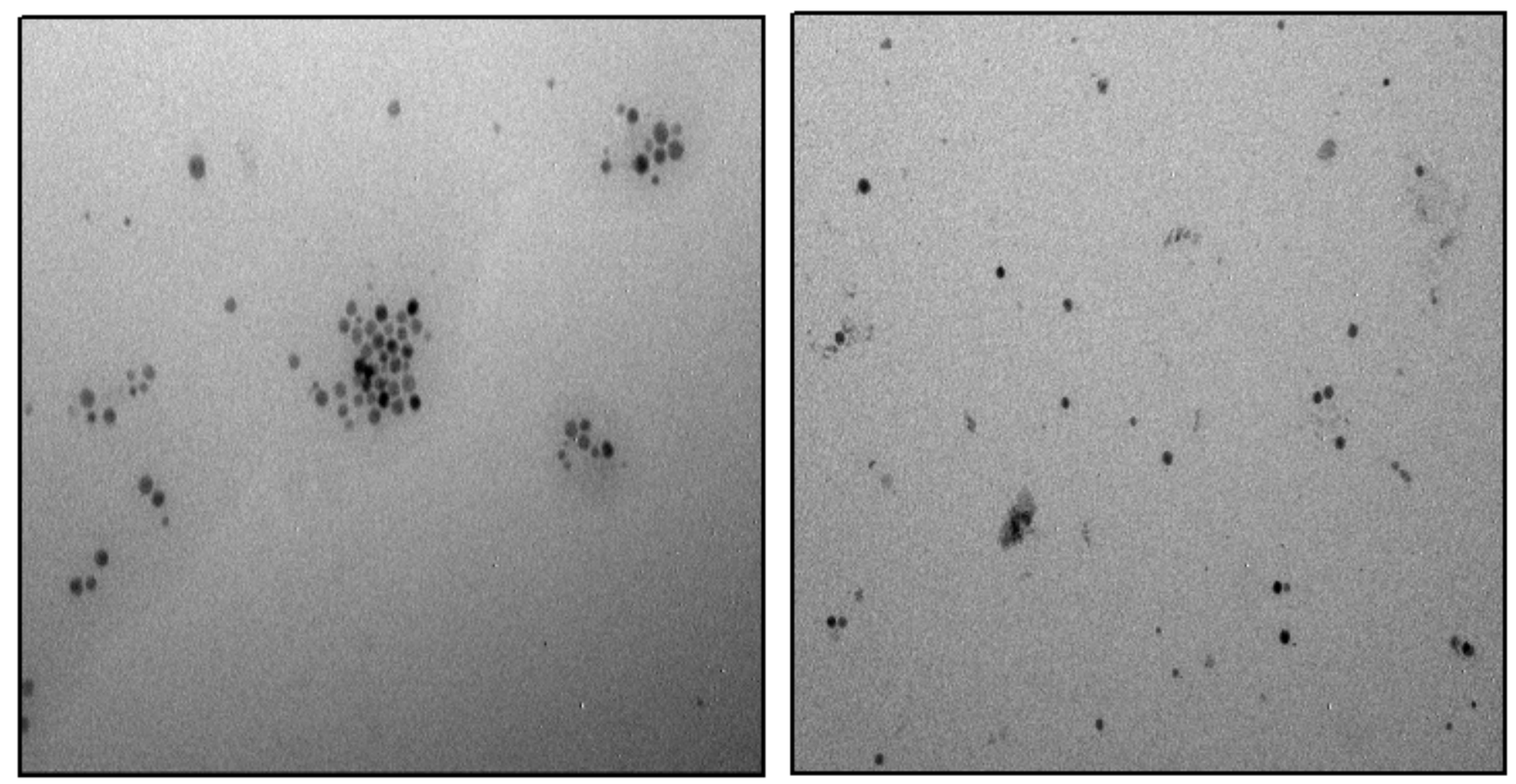

Figure 2

TEM photomicrographs of Vancomycin loaded cubosomes.

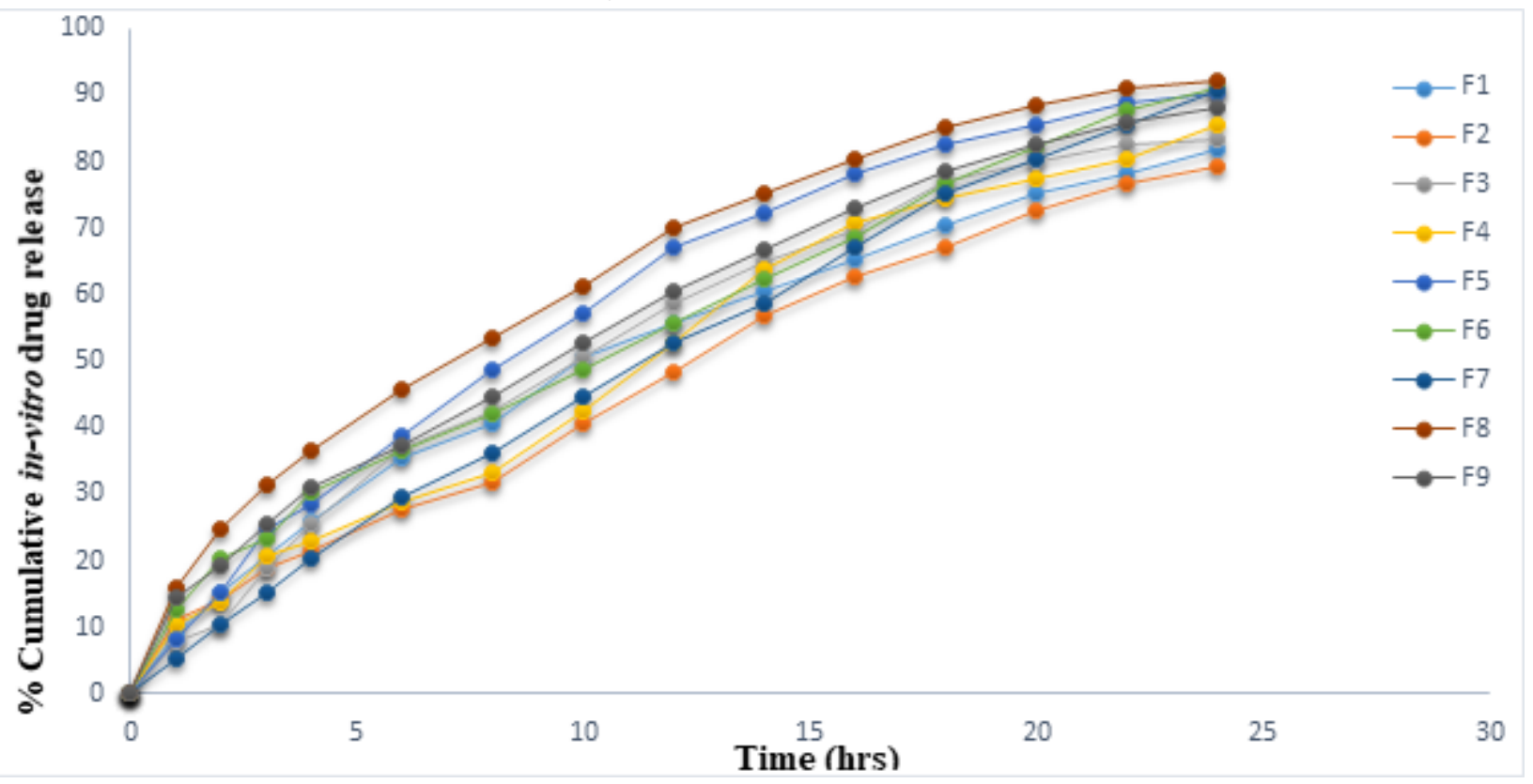

Figure 3

$\%$ Cumulative in-vitro drug release from VA-loaded cubosomes. 


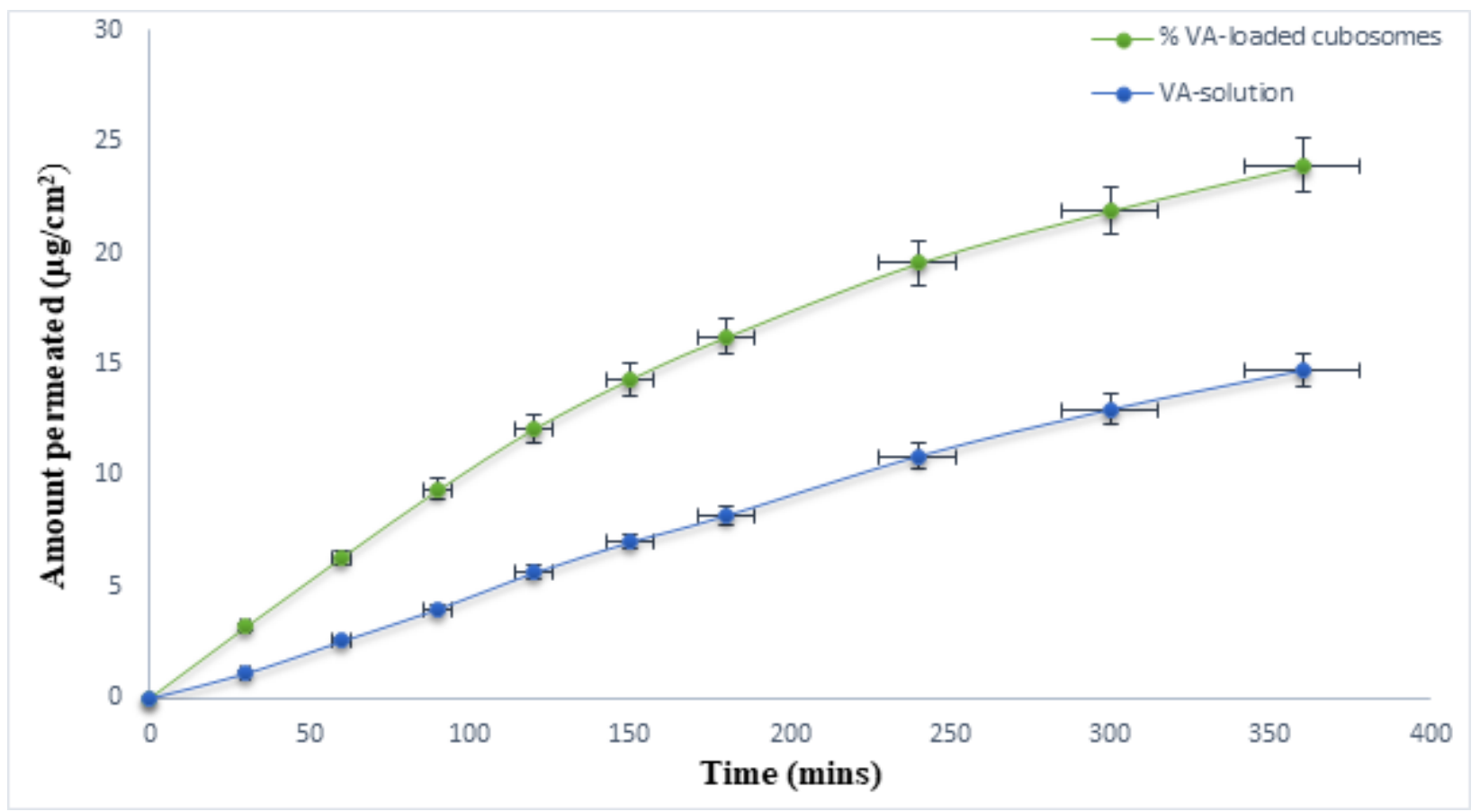

Figure 4

Transcorneal permeation profiles of VA-loaded cubosomes and VA-solution using excised rabbit corneas.
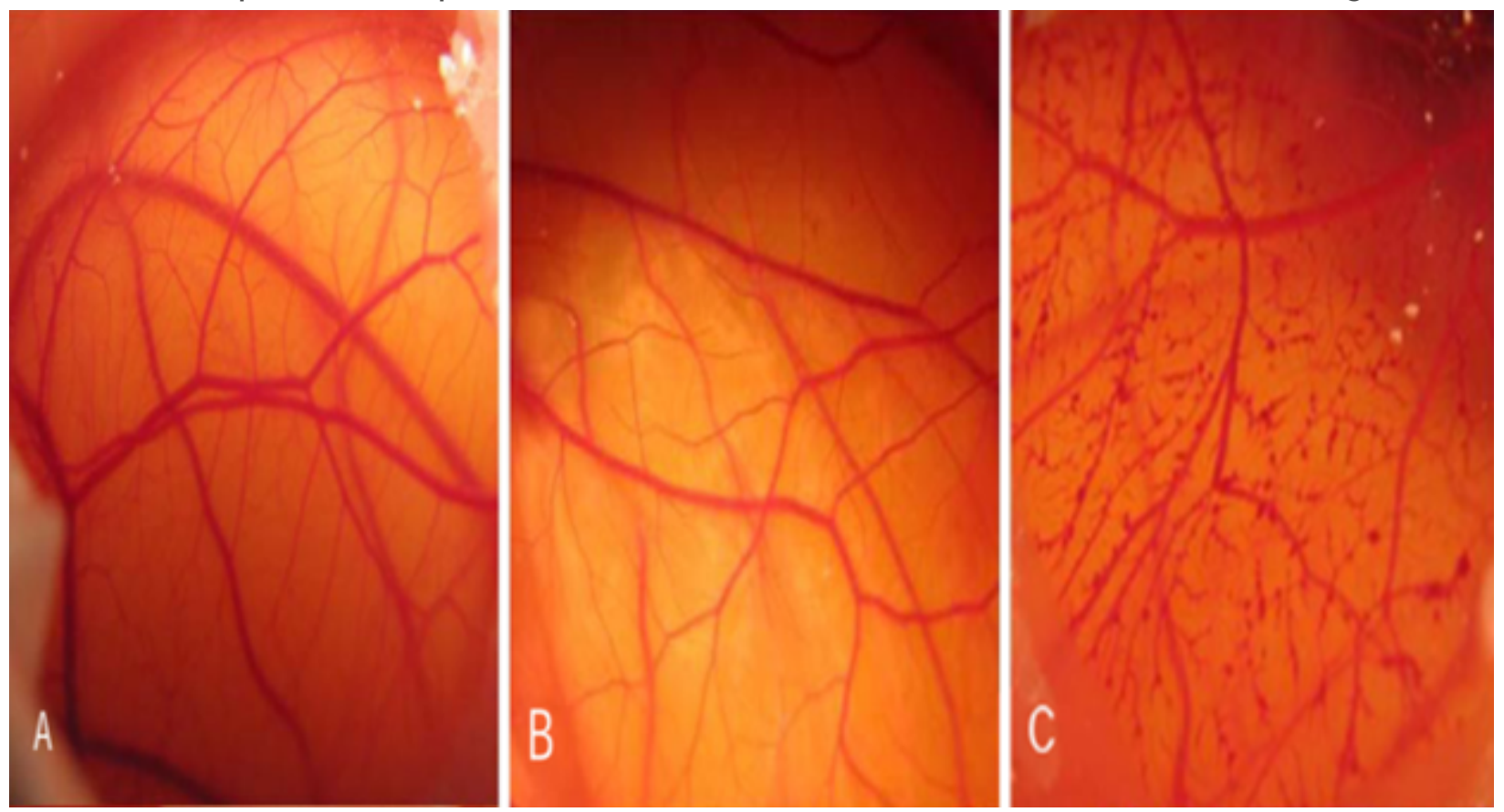

\section{Figure 5}

Photographs illustrating (A) Normal saline (negative control), (B) VA-loaded cubosomes and (C) $\mathrm{NaOH}$ (positive control). 


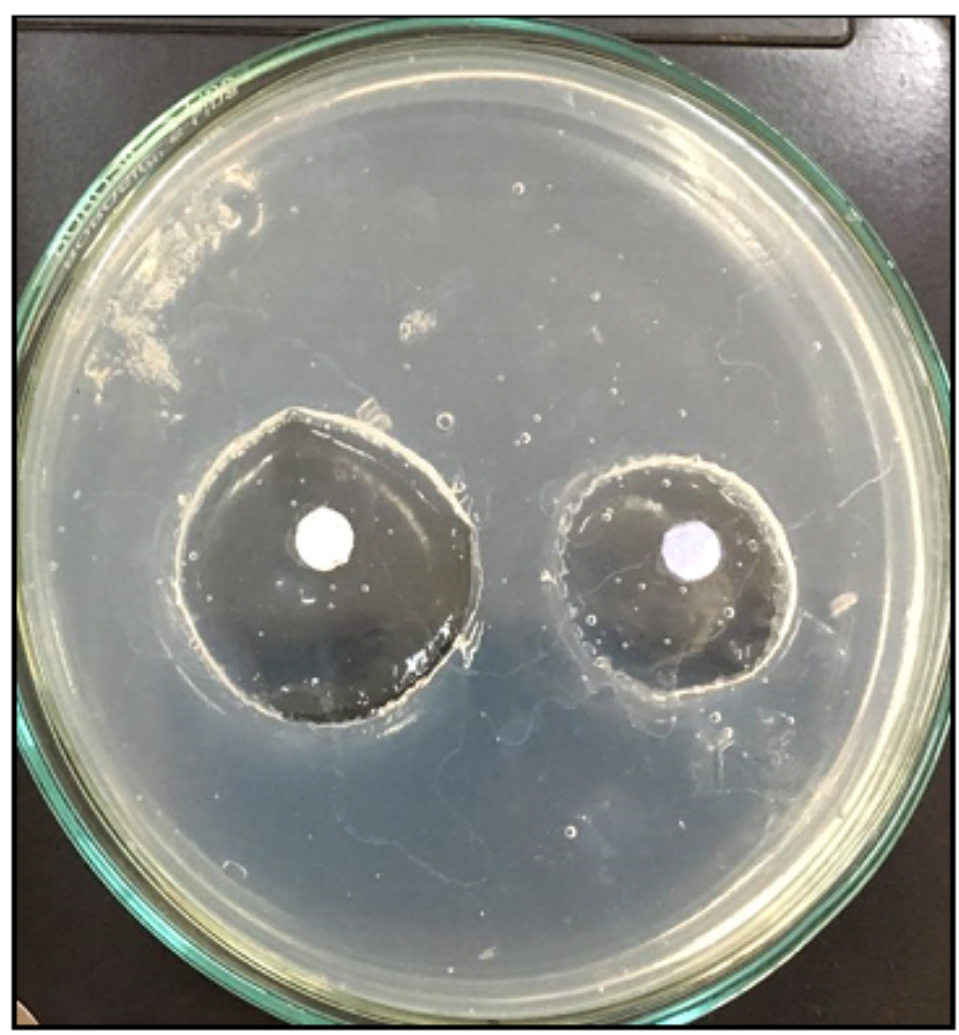

(a)

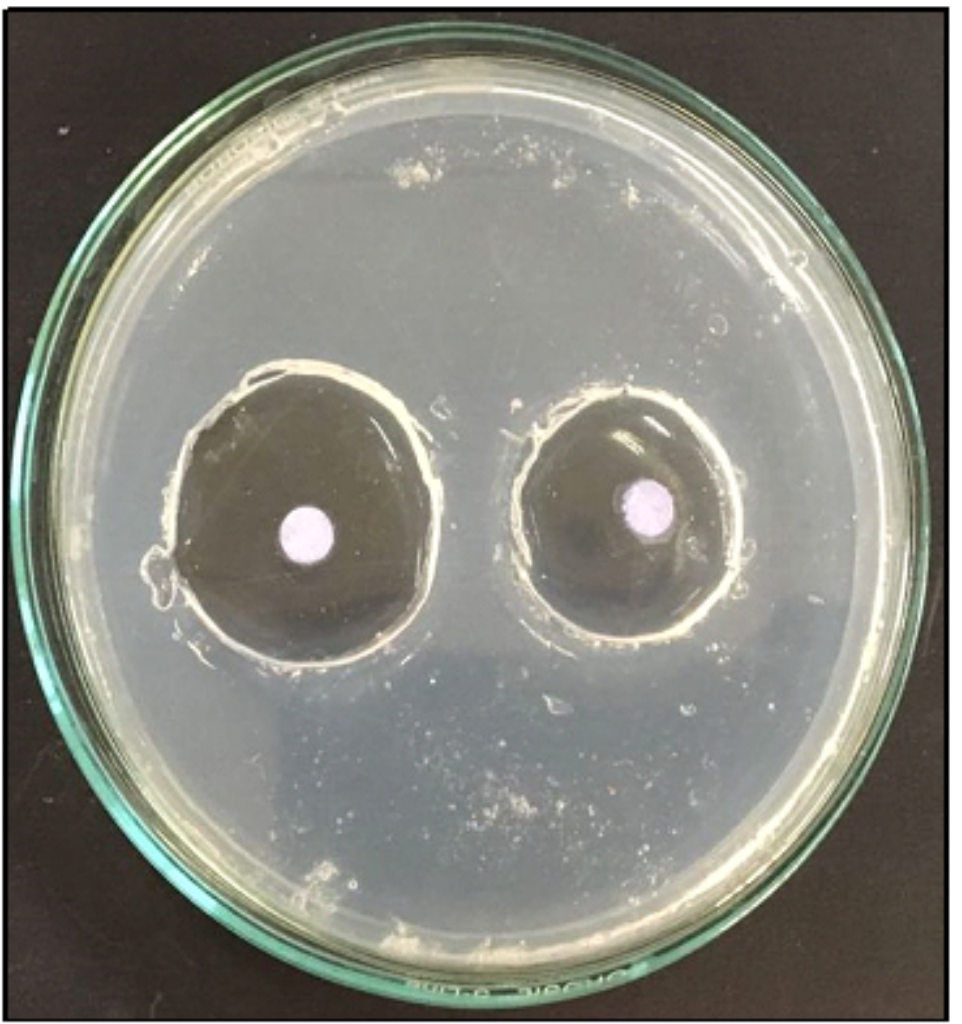

(b)

Figure 6

Images of antibacterial activity of VA-loaded cubosomes (a) inhibition against Staphylococcus Aureus and (b) inhibition against Pseudomonas aeruginosa (i) Test (ii) Control 


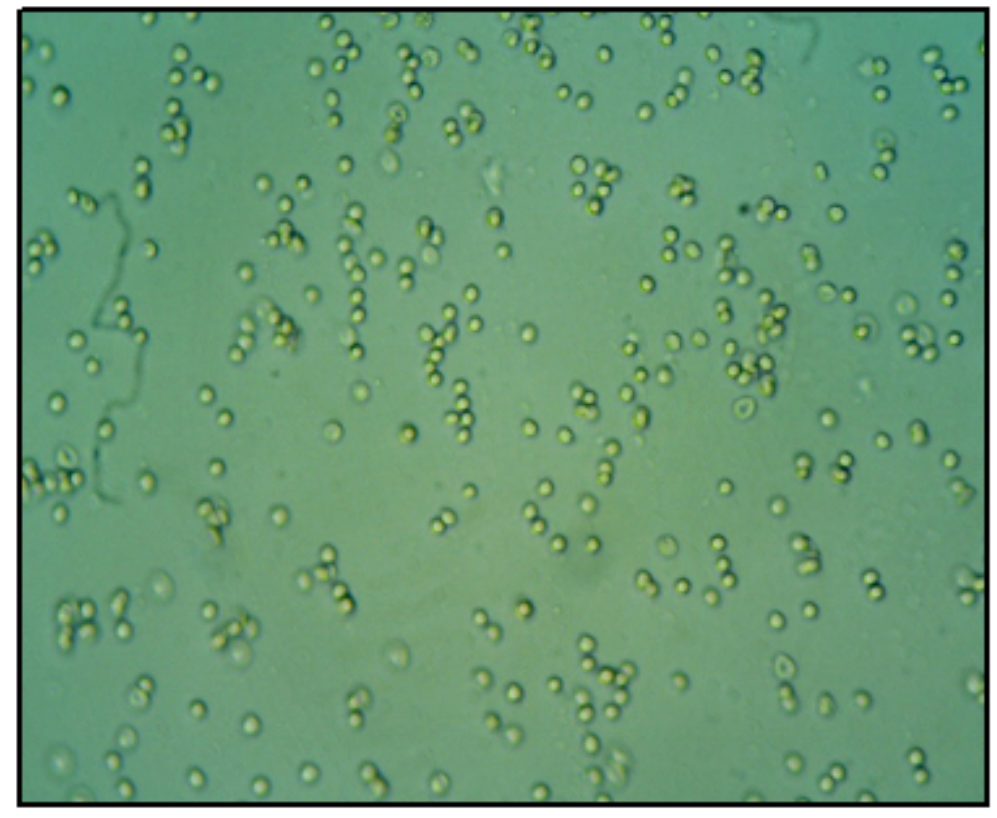

(a)

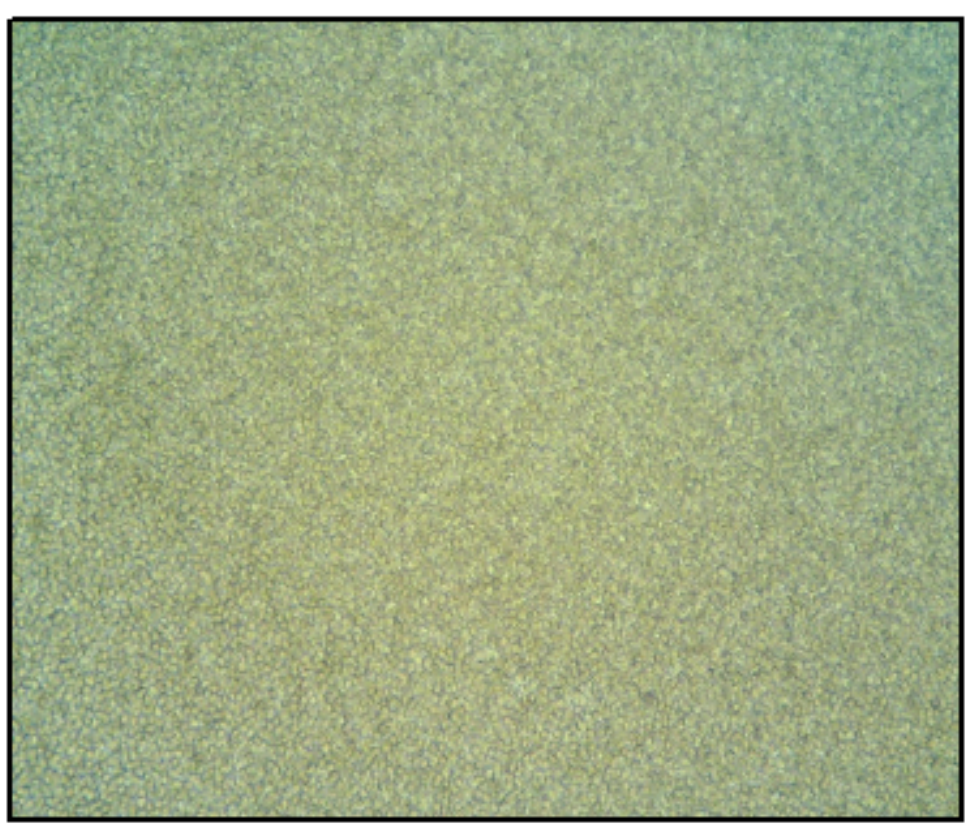

(c)

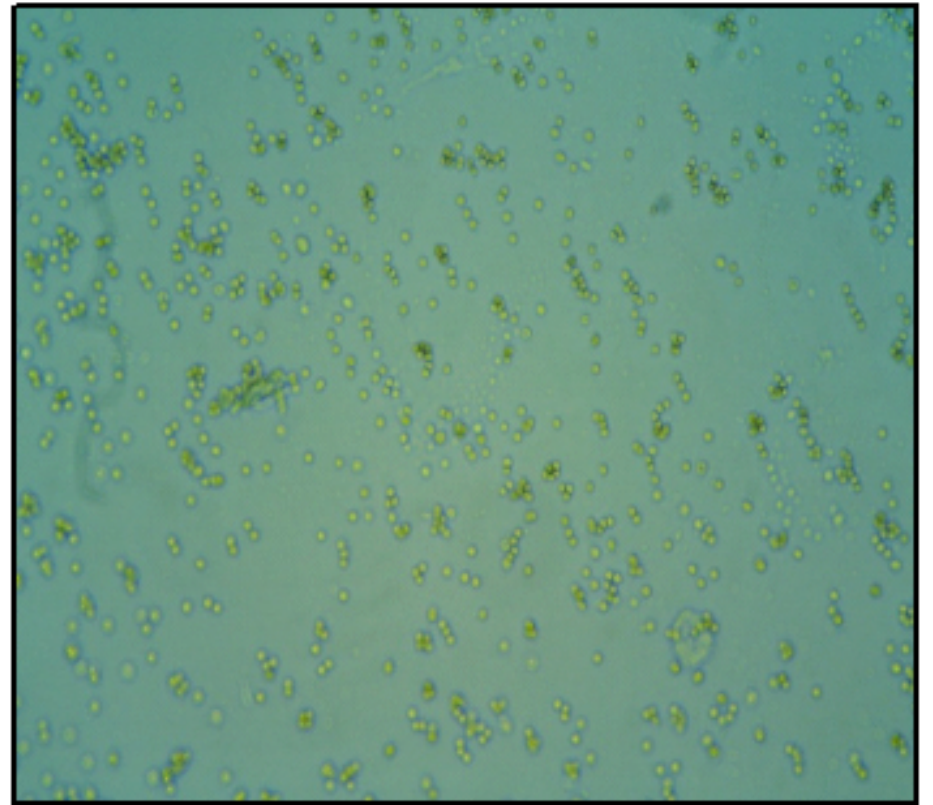

(b)

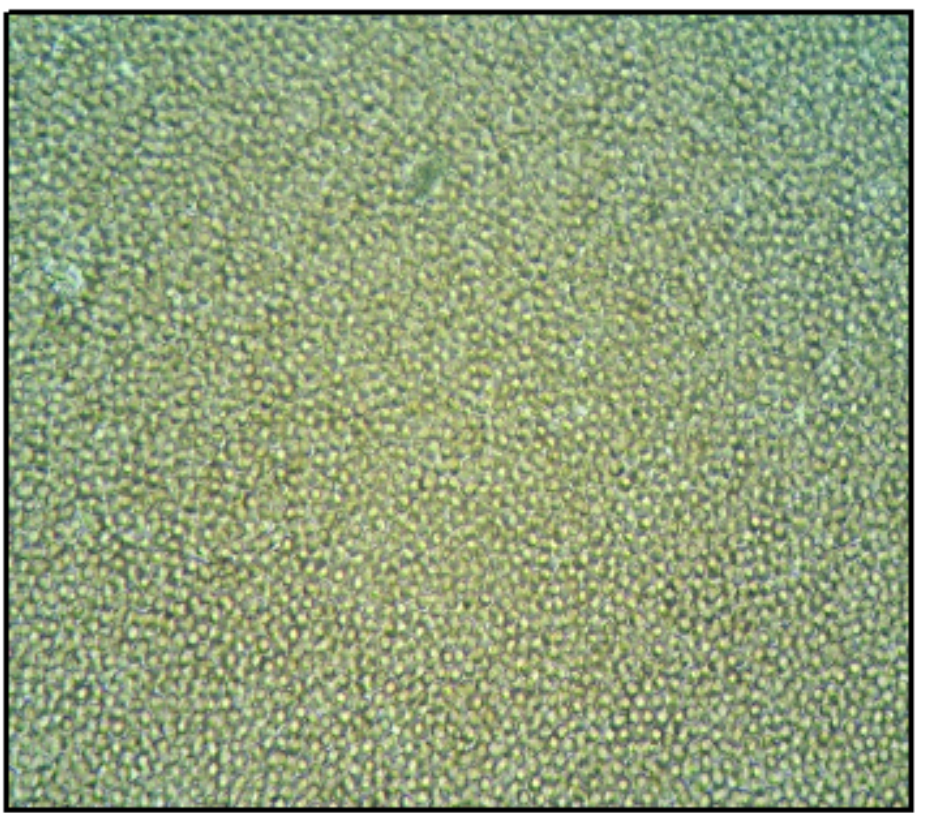

(d)

\section{Figure 7}

Microscopic images for isotonicity evaluation (a) Blood cells with VA-loaded cubosomes (b) Blood cells with isotonic solution (c) Blood cells with hypertonic solution (d) Blood cells with hypotonic solution 


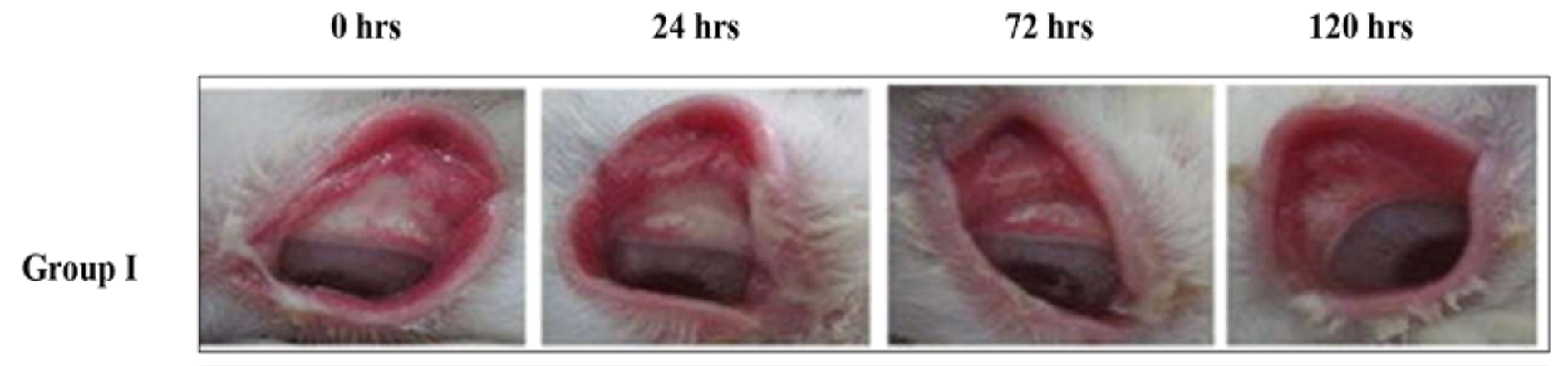

Group II

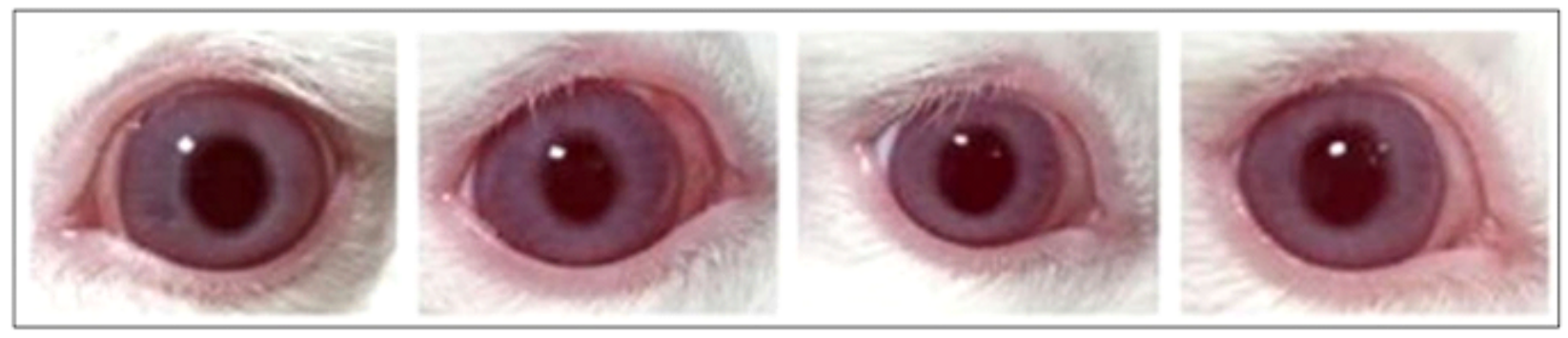

Group III
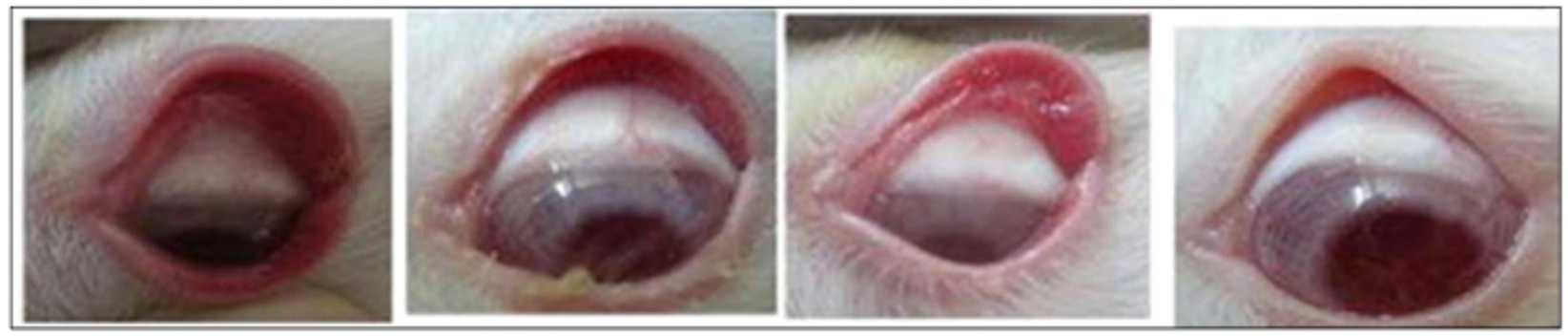

Figure 8

Images during treatment of infected rabbit eyes with bacterial keratitis after 0, 24, 72 and $120 \mathrm{hrs}$.

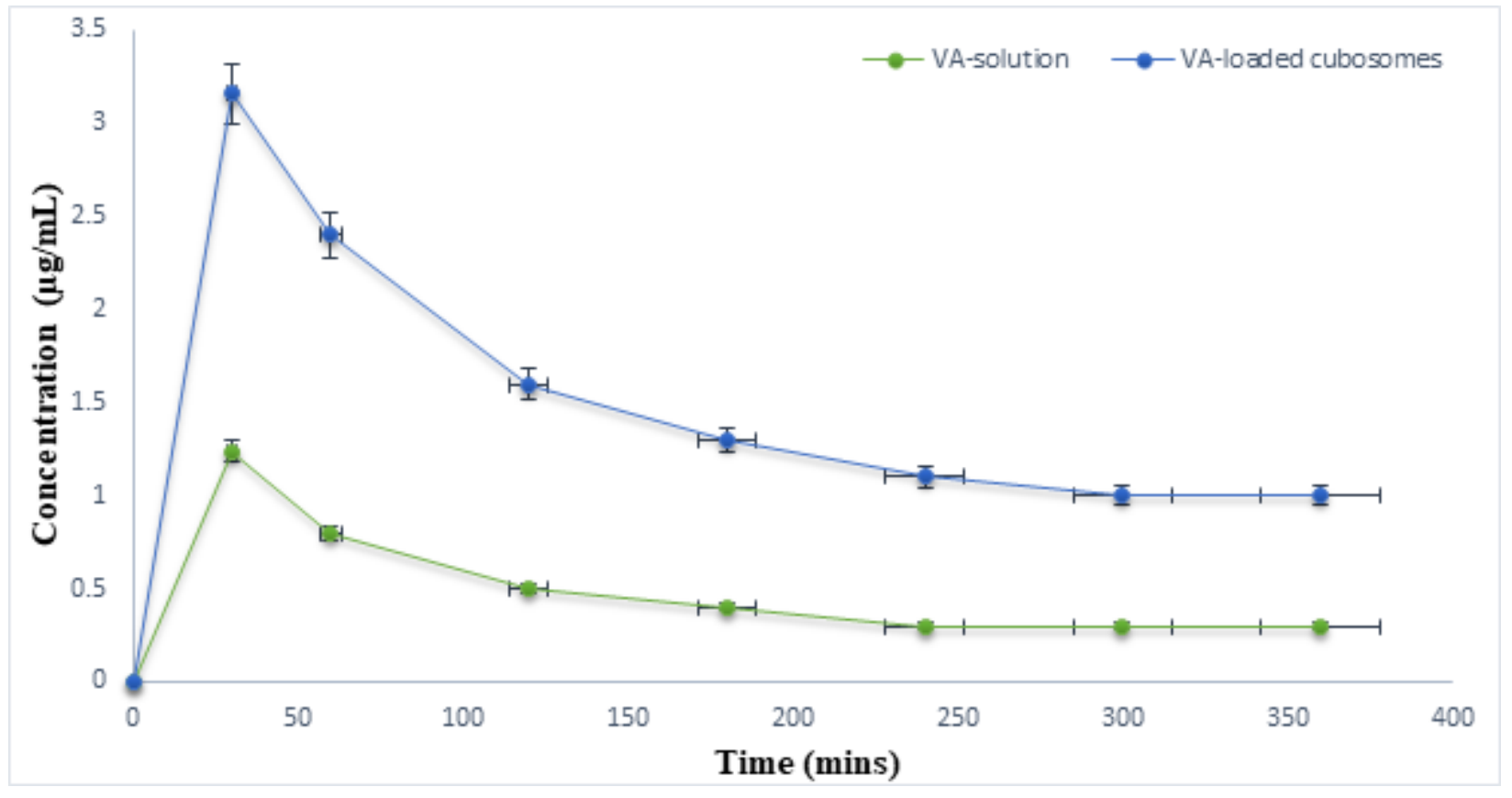

Figure 9 
Concentration-time profiles of vancomycin in aqueous humor after topical administration of VA-solution and VA-cubosomes.

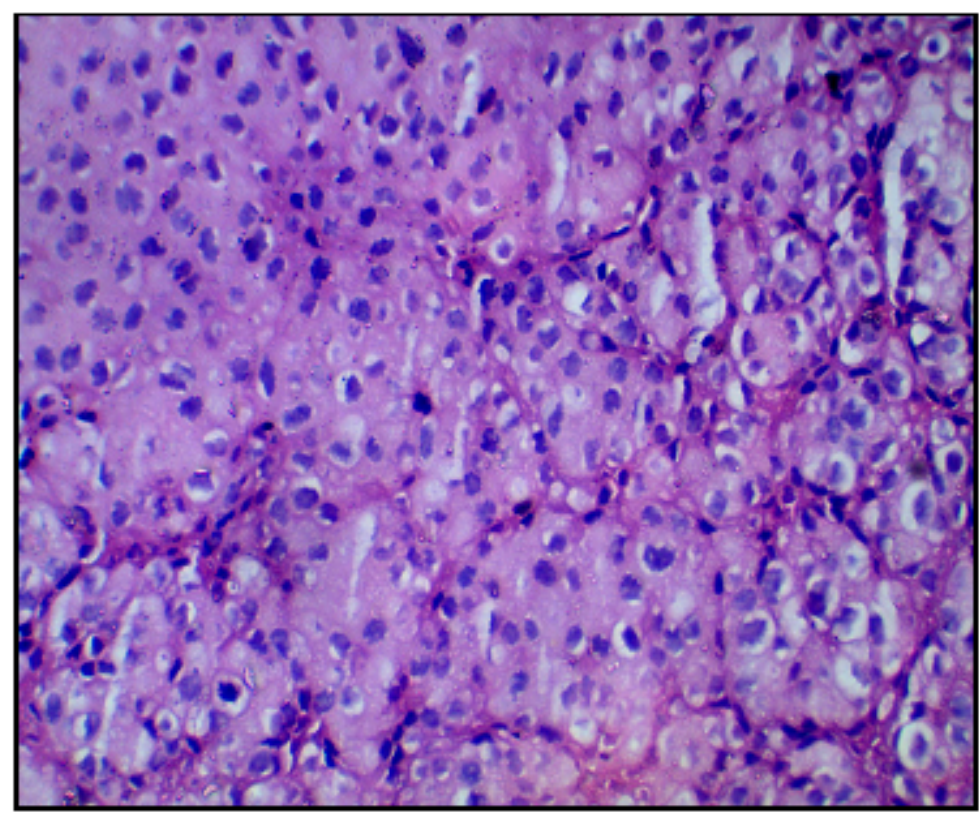

(a)

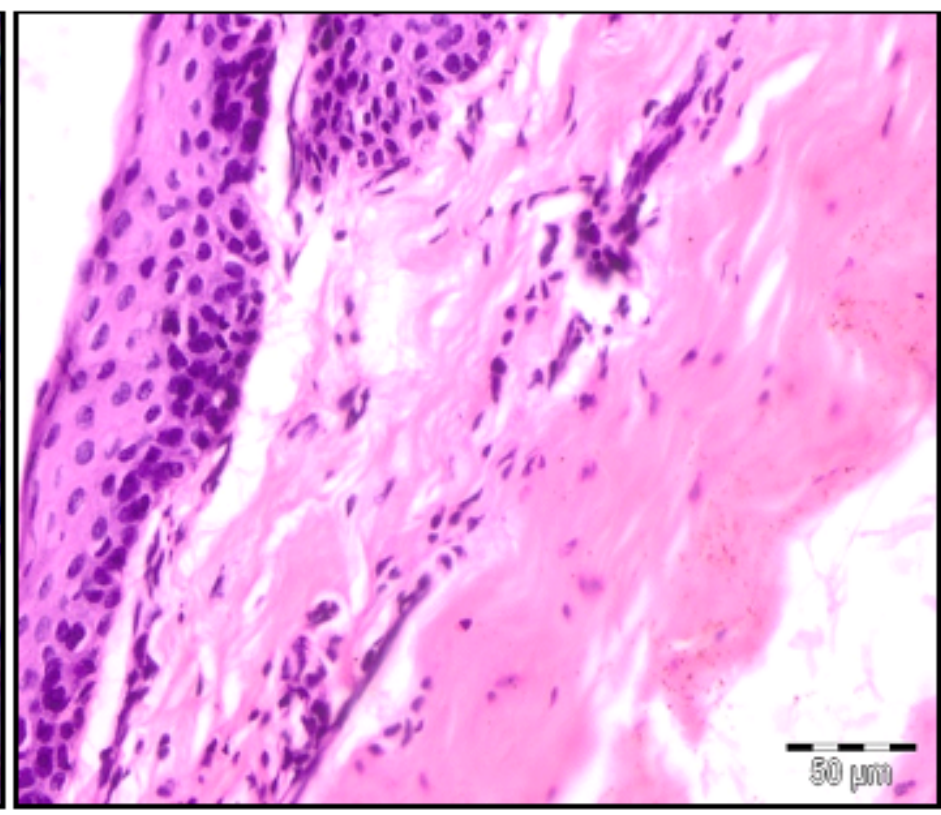

(b)

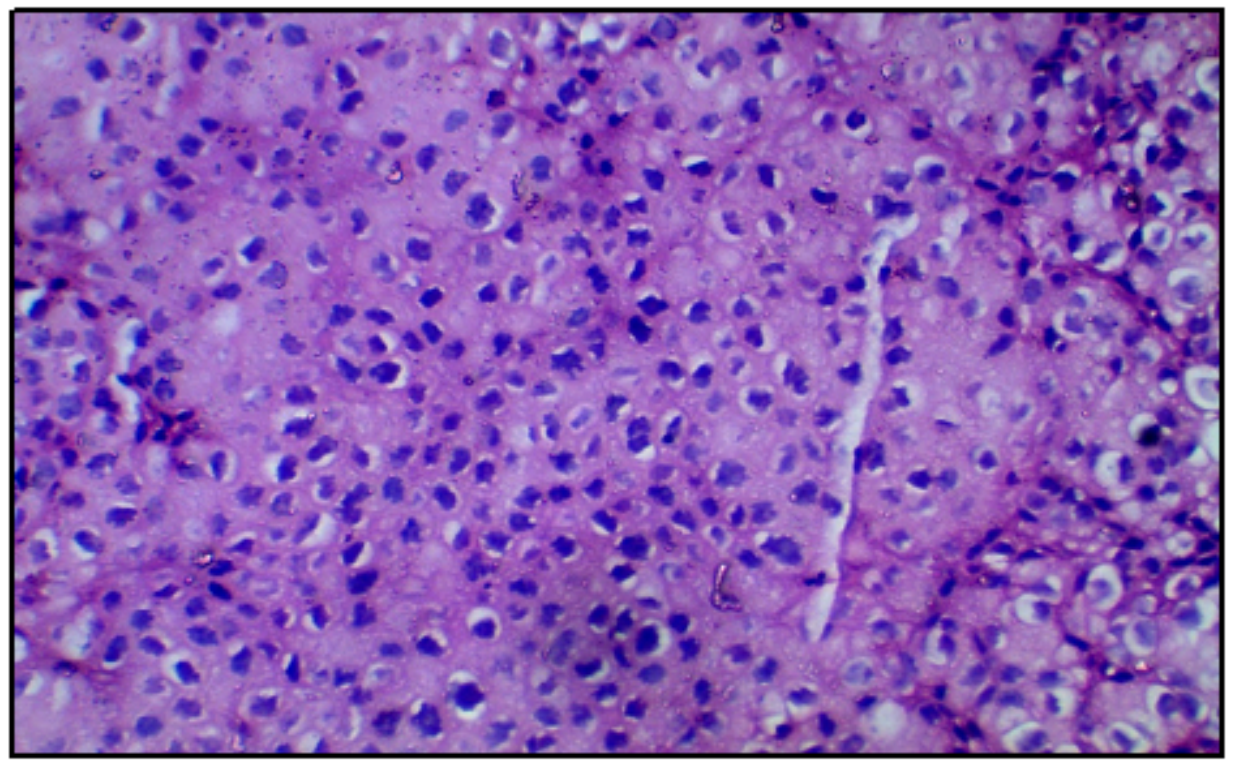

(c)

\section{Figure 10}

Comparative histopathological sections of rabbit cornea (a) negative control (untreated cornea) (b) Positive control (cornea treated with 0.1 \% SDS), (c) test sample (treated with VA-loaded cubosomes).

\section{Supplementary Files}

This is a list of supplementary files associated with this preprint. Click to download. 
- GraphicalAbstractPDF.pdf

Page 35/35 